\title{
Polarization, transmission, and small-angle scattering of light by a polymer film with elongated droplets of nematic liquid crystal
}

\author{
V.A. Loiko ${ }^{a *}$, A.V. Konkolovich ${ }^{a}$, A.A. Miskevich ${ }^{a}$, O.O. Prishchepa ${ }^{\text {b,c }}$, A.V. Shabanov ${ }^{\text {, }}$ \\ M.N. Krakhalev, ${ }^{\text {,c }}$, and V.Ya. Zyryanov ${ }^{b}$
}

\begin{abstract}
aStepanov Institute of Physics, National Academy of Sciences of Belarus, Niezalezhnastsi avenue 68-2 Minsk, 220072, Belarus

'Kirensky Institute of Physics, Federal Research Center "Krasnoyarsk Scientific Center", Siberian Branch, Russian Academy of Sciences, Academgorodok 50(38), Krasnoyarsk, 660036, Russia

'Institute of Engineering Physics and Radio Electronics, Siberian Federal University, Krasnoyarsk 660041, Russia

*E-mail: loiko@ifanbel.bas-net.by, http://loiko.org/, tel: +375 (17) 284-2894, fax: +375 (17) 2840879
\end{abstract}

\begin{abstract}
An optical-mechanical model has been developed to describe the transmittance, polarization and small-angle distribution of light scattered by an uniaxially-stretched polymer film containing the elongated ellipsoidal droplets of nematic liquid crystal. It is based on the approximations of FoldyTwersky, anomalous diffraction, and single scattering. The spectral dependences of transmittances and polarizing abilities of the polymer dispersed liquid crystal films have been analyzed as well as the small-angle intensity distribution and the polarization degree of scattered light depending on the film thicknesses, refractive index of polymer matrix, sizes of droplets, their anisometry parameters, concentration, polydispersity, and optical axes orientation. The optical characteristics of films with homogeneous and inhomogeneous interfacial anchoring at the surface of liquid crystal droplets have been considered. The transmittance and polarizing ability of the films have been studied as functions of the angular aperture of the system recording scattered light.
\end{abstract}

\section{Highlights:}

Polarization degree $>0.97$ of passed light is possible in spectral range $0.45-0.7 \mu \mathrm{m}$ Polarization degree $>0.95$ and transmittance $\approx 0.5$ are achieved at angular aperture $<3^{\circ}$ Stretched films with monodomain and bipolar structures are effective light polarizers

Keywords: polarization, polymer-dispersed liquid crystal, uniaxially stretched film, liquid crystal droplet, light transmittance, light scattering anisotropy.

\section{Introduction}


At present, polymer films with anisotropy of light absorption are most widely used as the polarizers [1-3]. A dichroism in such films is caused by the special additives into polymer matrix or by the anisotropy of own absorption of polymer macromolecules. The advantages of these materials are the compactness, low cost, and simple production technology. However, these polymer films are applicable in the laser and optical devices only at low power since a light absorption can result in the strong heating and following destruction of polymer matrix.

Polymer dispersed liquid crystal (PDLC) films, consisting of non-absorbing uniaxiallyelongated liquid crystal (LC) droplets within polymer matrix [4-10], used as a polarizers, allow one to increase significantly the limit power of the incident light in comparison with dichroic polarizers. PDLC films can polarize a light with the power density of incident light up to $2 \mathrm{kWt} / \mathrm{cm}^{2}$ [10]. They effectively polarize a light in the entire transparency region (visible and near IR) of the components used, while the dichroic polarizers can do that only in the dichroic band of own or impurity absorption. Besides, PDLC films allow modulating the intensity, polarization and phase of light by applying the electric or magnetic field. They are particularly promising in the collimated laser devices and projection systems.

Recently, a new approach has been realized to control the optical response of PDLC film in the light-scattering mode, which is based on the surface anchoring transitions caused by ionic surfactant [11]. Unidirectional mechanical stretching of PDLC film doped with the ionic surfactants allows obtaining the LC director configuration (distribution of local optical axes) within the droplet volume close to homogeneous (monodomain) $[12,13]$. Thus, it gives an opportunity to achieve simultaneously the limit polarization characteristics for unpolarized incident light: the coherent transmission coefficient equal to 0.5 and the polarization degree of forward-transmitted light equal to \pm 1 in the wide spectral range [14].

In this work we have studied the spectral dependences of the coefficients of coherent (directed) transmission and the polarization degree of forward-transmitted light, the angular distribution and polarization of light scattered in small angles by the stretched PDLC films with homogeneous (without surfactants) and inhomogeneous (with surfactants) surface anchoring. It has been considered the films consisting of ellipsoidal LC droplets with: (i) the bipolar intrinsic structure formed under homogeneous tangential anchoring; (ii) the radial structure formed under homogeneous homeotropic (normal) anchoring; (iii) the monodomain structure raised due to inhomogeneous surface anchoring $[12,13]$ (Fig. 1).
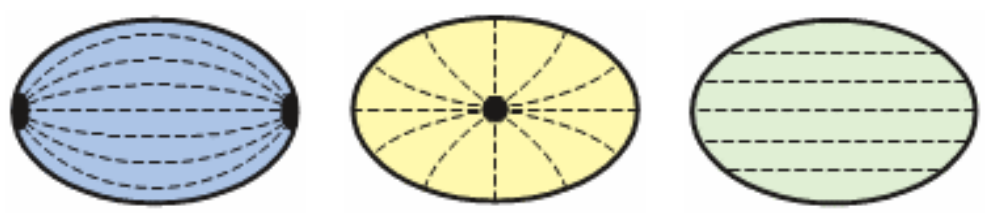
Figure 1. Orientational structures within ellipsoidal nematic droplets: bipolar (a), radial (b), and homogeneous monodomain (c). Director configurations are presented by dashed lines. Surface point defects (boojums) are indicated by black semicircles (a); bulk point defect (hedgehog) is shown by black circle (b).

The transmittance, the small-angle distribution of scattered light and the polarizing ability of the film containing the ellipsoidal monodomain LC droplets have been analyzed as functions of angular aperture of the optical system.

\section{Optical-mechanical model to describe the characteristics of scattered light. Basic equations}

Let us consider a PDLC layer of thickness $l$ placed in the $(y, z)$ plane of laboratory coordinate system ( $x y z)$, normally illuminated along $x$ axis by unpolarized monochromatic light (Fig. 2).

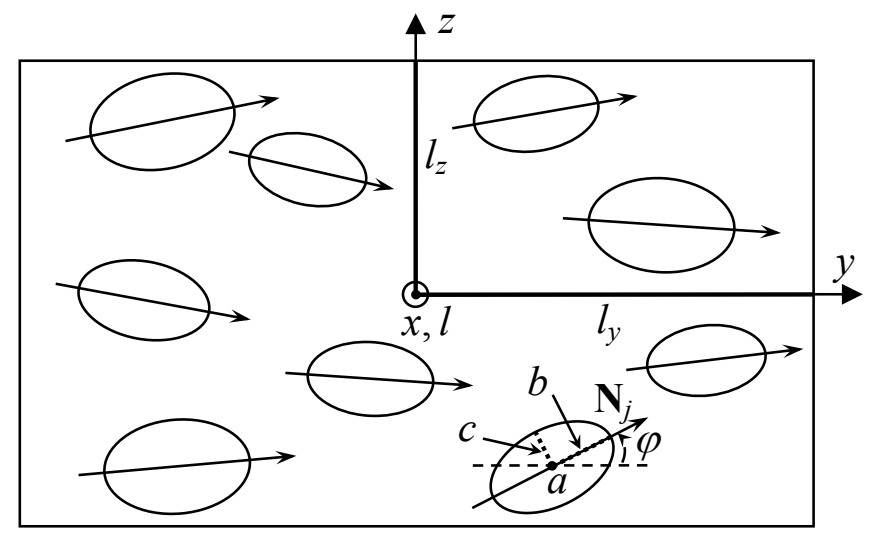

Figure 2. Scheme of the PDLC layer structure under stretching along $y$ axis of the laboratory coordinate system $(x, y, z)$. The $(y, z)$ plane coincides with the layer-front surface. The $x$ axis is directed along the normal to PDLC layer; $\varphi$ is the orientation angle of optical axis $\mathbf{N}_{j}$ of an individual droplet; $l$ and $l_{y}, l_{z}$ are the thicknesses of stretched layer and its linear sizes along $y, z$ axes; $a, b, c$ are the semiaxes of elongated ellipsoidal droplets. The $a$ semiaxes are oriented along $x$ axis and $b, c$ semiaxes are aligned in the $(y, z)$ layer plane. The layer is normally illuminated by unpolarized light along $x$ axis.

Initially, the undeformed polymer matrix contains the polydisperse spherical (or spheroidal) LC droplets with circular section. Their optical axes are randomly oriented in $(y, z)$ plane. The mechanical stretching of PDLC film along $y$ axis results in the deformation of spheroidal droplets into the ellipsoidal ones with the $a, b, c$ semiaxes and partially-oriented structure of optical axes $\mathbf{N}_{j}$, $j=1,2, \ldots, N$, where $N$ is a number of droplets in the layer. The major semiaxes $b$ of droplets and their optical axes $\mathbf{N}_{j}$ are preferably oriented along the stretching axis $y$, the $c$ semiaxes are aligned 
within $(y, z)$ layer plane, the $a$ semiaxes are directed along the $x$ normal. The semiaxes of spherical droplets before stretching are designated by $a_{0}=b_{0}=c_{0}$. For spheroidal droplets $a_{0}<b_{0}=c_{0}$.

\subsection{Coefficient of coherent (directed) transmission of the stretched PDLC film and the polarization degree of forward-transmitted light}

Using the Foldy-Twersky approximation [14-19], we can write the expressions for the coherent (directed) transmission coefficient $T_{n p}^{c}$ and polarization degree $P_{c}$ of the forwardtransmitted light (the polarizing ability of layer for forward-transmitted light):

$$
\begin{gathered}
T_{n p}^{c}=\frac{T_{\|}^{c}+T_{\perp}^{c}}{2}, \\
P_{c}=\frac{T_{\perp}^{c}-T_{\|}^{c}}{T_{\|}^{c}+T_{\perp}^{c}}, \\
T_{\|, \perp}^{c}=\exp \left(-\gamma_{2,1} l\right), \\
\gamma_{2,1}=\frac{3}{4} c_{v} \frac{\left\langle a^{2}\right\rangle}{\left\langle a^{3}\right\rangle}\left\{\left\langle Q_{e}\right\rangle \frac{1 \pm S_{2 f}}{2}+\left\langle Q_{o}\right\rangle \frac{1 \mp S_{2 f}}{2}\right\}, \\
\left\langle a^{2}\right\rangle=\int_{0}^{\infty} a^{2} P(a) d a, \\
\left\langle a^{3}\right\rangle=\int_{0}^{\infty} a^{3} P(a) d a, \\
\left\langle Q_{e, o}\right\rangle=\frac{4\left\langle\operatorname{Re} S_{2,1}^{0}\right\rangle}{k^{2} \varepsilon_{y} \varepsilon_{z}\left\langle a^{2}\right\rangle}, \\
\left\langle\operatorname{Re} S_{2,1}^{0}\right\rangle=\int_{0}^{\infty} \operatorname{Re} S_{2,1}\left(\theta_{s}=0, a, b=\varepsilon_{y} a, c=\varepsilon_{z} a\right) P(a) d a, \\
\sin \left(2 \varphi_{m}\right) \\
2 \varphi_{m}
\end{gathered}
$$

Here $T_{\|}{ }^{c}$ and $T_{\perp}{ }^{c}$ are the coefficients of coherent transmission for the components of incident light polarized parallel and orthogonal to the stretching axis; $\gamma_{2}$ and $\gamma_{1}$ are the corresponding extinction coefficients of the layer; $c_{v}$ is the volume filling factor of layer (the ratio of volume of all LC droplets to the volume of PDLC layer); $\left\langle Q_{e}\right\rangle$ and $\left\langle Q_{o}\right\rangle$ are the extinction efficiency factors [20] for the extraordinary and ordinary waves, averaged over the droplet sizes; $S_{2 f}$ is the two-dimensional order parameter of PDLC layer; $k=2 \pi n_{p} / \lambda, \lambda$ is the wavelength of incident light, $n_{p}$ is the refractive index of polymer matrix; $\varepsilon_{y}=b / a$ and $\varepsilon_{z}=c / a$ are the parameters of droplet's anisometry; $\left\langle\operatorname{Re} S_{2,1}^{0}\right\rangle$ are 
the averaged real parts of the diagonal elements of amplitude scattering matrix for the strictly forward direction of scattering [14]; the angle brackets $\langle\ldots\rangle$ mean the average over the $a$ semiaxis of droplets along the film normal; $P(a)$ is the normalized probability density of $a$ semiaxes distribution along the film normal; $\varphi_{m}$ is the maximal angle of droplet optical axes deviation relatively to the stretching axis. At $\varphi_{m}=0$ the optical axes of droplets are entirely oriented along the stretching axis $\left(S_{2 f}=1\right)$. At $\varphi_{m}=\pi$ they are oriented randomly $\left(S_{2 f}=0\right)$. The partial orientation occurs at $0<\varphi_{m}<\pi$ $\left(S_{2 f}<1\right)$.

The Eqs. (6)-(9) were obtained at the same anisometry parameters $\varepsilon_{y}$ and $\varepsilon_{z}\left(\varepsilon_{y}=b / a=\right.$ const, $\varepsilon_{z}=c / a=$ const) for all droplets assuming the uniform distribution of the optical axes $\mathbf{N}_{j}$ orientation in the $(y, z)$ plane.

At stretching, the layer thickness $l$ and the semiaxes $a, b, c$ of droplets are related by the expressions [6,14]:

$$
l=l_{0} p^{-B}, a=a_{0} p^{-B}, b=c_{0} p, c=c_{0} p^{-A},
$$

where $p$ is the stretching factor ( $p=l_{y} / l_{y 0}, l_{y 0}$ is the layer length along $y$-axis before stretching); $l_{0}$ is the layer thickness before stretching; constants $A$ and $B$ are determined by mechanical properties of the used polymer, $A+B=1$ [6]. If initially the LC droplets are spherical, then $A=B=0.5$. For the initially spheroidal droplets: $A \approx 0.4$ and $B \approx 0.6$.

\subsection{Angular distribution of intensity and polarization degree of incoherently (diffusely) scattered light}

Let the layer is illuminated by unpolarized light. Then the intensity $I_{n p}^{i n c}$ and polarization degree $P_{\text {inc }}$ of incoherently (diffusely) scattered light, the parallel $I_{\|}{ }^{i n c}$ and orthogonal $I_{\perp}{ }^{i n c}$ to the stretching axis components of scattered light intensity are determined as follows:

$$
\begin{aligned}
& I_{n p}^{i n c}=\frac{1}{2}\left(I_{\|}^{i n c}+I_{\perp}^{i n c}\right), \\
& P_{i n c}=\frac{I_{\perp}^{i n c}-I_{\|}^{i n c}}{I_{\|}^{i n c}+I_{\perp}^{i n c}}, \\
& I_{\|, \perp}^{i n c}=\left(I_{v v}^{i n c}+I_{v h}^{i n c}\right)_{\alpha=0, \pi / 2} .
\end{aligned}
$$

Here $I_{v v}^{i n c}$ and $I_{v h}^{i n c}$ are $v v$ - and $v h$-components of the intensity of scattered light with the parallel ( $v v$ component) and orthogonal ( $v h$-component) polarizations relatively to the polarization plane of incident wave; $\alpha$ is the polarization angle (angle between the polarization plane of incident wave and the stretching axis $y$ ) $[21,22]$. Relation (13) is obtained by averaging $I_{v v}^{i n c}$ and $I_{v h}^{i n c}$ over the polarization angle $\alpha$ with a uniform $\alpha$-distribution ranging from 0 to $2 \pi$ [22]. 
If the filling factor $c_{v}$ of the layer is low $\left(c_{v}<0.2\right)$, then, using the single scattering approximation [23] for $I_{v v}^{i n c}$ and $I_{v h}^{i n c}$ components of light intensity (entering in Eq. (13)), we obtain:

$$
I_{v v, v h}^{i n c}\left(\theta_{s}, \varphi_{s}\right)=C \frac{3}{4} \frac{l\left\langle a^{2}\right\rangle}{\varepsilon_{y} \varepsilon_{z}\left\langle a^{3}\right\rangle} \frac{c_{v}}{k^{2}\langle\sigma\rangle}\left\langle\left|f_{v v, v h}\left(\theta_{s}, \varphi_{s}\right)\right|^{2}\right\rangle_{a, \mathbf{N}_{j}},
$$

where $\theta_{s}$ and $\varphi_{s}$ are the polar and azimuthal scattering angles [22,24];C=|E $E_{i}^{2} \mid A / R^{2}, E_{i}$ is the amplitude of incident wave, $A$ is the area of the illuminated part of the layer; $R$ is the distance from layer to the observation point; $\sigma=\pi b c$ is the section of droplet in the $y z$ layer plane; $f_{v v}\left(\theta_{s}, \varphi_{s}\right)$ and $f_{v h}\left(\theta_{s}, \varphi_{s}\right)$ are $v v$ - and $v h$-components of vector amplitude scattering function of the individual LC droplet $[21,22,24]$; the angle brackets $\langle\ldots\rangle_{a, \mathbf{N}_{j}}$ mean averaging over the size $a$ and orientation of optical axes $\mathbf{N}_{j}$ of droplets.

Assuming independence of the distributions of droplets over the size and orientation of their optical axes, we have:

$$
\begin{aligned}
& \left\langle\left|f_{v v}\right|^{2}\right\rangle_{a, \mathbf{N}_{j}}=\left\langle\left|S_{2}\right|^{2}\right\rangle \overline{\cos ^{4}\left(\alpha-\varphi_{s}-\varphi_{d}\right)}+\left\langle\left|S_{1}\right|^{2}\right\rangle \overline{\sin ^{4}\left(\alpha-\varphi_{s}-\varphi\right)}+ \\
& +\frac{1}{4}\left\langle\left\{S_{1} S_{2}^{*}+S_{2} S_{1}^{*}+\left(S_{3}+S_{4}\right)\left(S_{3}^{*}+S_{4}^{*}\right)\right\}\right\rangle \overline{\sin ^{2} 2\left(\alpha-\varphi_{s}-\varphi\right)}+ \\
& +\frac{1}{8}\left\langle\left\{\left(S_{3}+S_{4}\right) S_{2}^{*}+S_{2}\left(S_{3}^{*}+S_{4}^{*}\right)\right\}\right\rangle\left[\overline{\sin 2\left(\alpha-\varphi_{s}-\varphi\right)}+\overline{\sin 4\left(\alpha-\varphi_{s}-\varphi\right)}\right]+ \\
& +\frac{1}{8}\left\langle\left\{\left(S_{3}+S_{4}\right) S_{1}^{*}+S_{1}\left(S_{3}^{*}+S_{4}^{*}\right)\right\}\right\rangle\left[2 \overline{\sin 2\left(\alpha-\varphi_{s}-\varphi\right)}-\overline{\sin 4\left(\alpha-\varphi_{s}-\varphi\right)}\right] \\
& \left\langle\left|f_{v h}\right|^{2}\right\rangle_{a, \mathbf{N}_{j}}=\left\langle\left|S_{3}\right|^{2}\right\rangle \overline{\sin ^{4}\left(\alpha-\varphi_{s}-\varphi_{d}\right)}+\left\langle\left|S_{4}\right|^{2}\right\rangle \overline{\cos ^{4}\left(\alpha-\varphi_{s}-\varphi\right)}+ \\
& +\frac{1}{4}\left\langle\left\{\left(S_{2}-S_{1}\right)\left(S_{2}^{*}-S_{1}^{*}\right)-S_{4} S_{3}^{*}-S_{3} S_{4}^{*}\right\}\right\rangle \overline{\sin ^{2} 2\left(\alpha-\varphi_{s}-\varphi\right)}- \\
& -\frac{1}{8}\left\langle\left\{\left(S_{2}-S_{1}\right) S_{4}^{*}+S_{4}\left(S_{2}^{*}-S_{1}^{*}\right)\right\}\right\rangle\left[2 \overline{\sin 2\left(\alpha-\varphi_{s}-\varphi\right)}+\overline{\sin 4\left(\alpha-\varphi_{s}-\varphi\right)}\right]+ \\
& +\frac{1}{8}\left\langle\left\{\left(S_{2}-S_{1}\right) S_{3}^{*}+S_{3}\left(S_{2}^{*}-S_{1}^{*}\right)\right\}\right\rangle\left[2 \overline{\sin 2\left(\alpha-\varphi_{s}-\varphi\right)}-\overline{\sin 4\left(\alpha-\varphi_{s}-\varphi\right)}\right]
\end{aligned}
$$

where $S_{j}=S_{j}\left(\theta_{s}, \varphi_{s}\right)$ are the elements of the amplitude scattering matrix of the individual LC droplet $(j=1,2,3,4)[22,24-26]$.

For uniform distribution of droplet axes orientation over the angle $\varphi$, we can write:

$$
\begin{gathered}
\overline{\cos ^{4}\left(\alpha-\varphi_{s}-\varphi_{d}\right)}=\frac{3}{8}+\frac{1}{2} \cos \left(2\left(\alpha-\varphi_{s}\right)\right) \operatorname{sinc}\left(2 \varphi_{m}\right)+\frac{1}{8} \cos \left(4\left(\alpha-\varphi_{s}\right)\right) \operatorname{sinc}\left(4 \varphi_{m}\right), \\
\overline{\sin ^{4}\left(\alpha-\varphi_{s}-\varphi_{d}\right)}=\frac{3}{8}-\frac{1}{2} \cos \left(2\left(\alpha-\varphi_{s}\right)\right) \operatorname{sinc}\left(2 \varphi_{m}\right)+\frac{1}{8} \cos \left(4\left(\alpha-\varphi_{s}\right)\right) \operatorname{sinc}\left(4 \varphi_{m}\right), \\
\overline{\sin ^{2}\left(2\left(\alpha-\varphi_{s}-\varphi_{d}\right)\right)}=\frac{1}{2}\left(1-\cos \left(4\left(\alpha-\varphi_{s}\right)\right) \operatorname{sinc}\left(4 \varphi_{m}\right)\right),
\end{gathered}
$$




$$
\begin{aligned}
& \overline{\sin 2\left(\alpha-\varphi_{s}-\varphi_{d}\right)}=\sin \left(2\left(\alpha-\varphi_{s}\right)\right) \operatorname{sinc}\left(2 \varphi_{m}\right), \\
& \overline{\sin \left(4\left(\alpha-\varphi_{s}-\varphi_{d}\right)\right)}=\sin \left(4\left(\alpha-\varphi_{s}\right)\right) \operatorname{sinc}\left(4 \varphi_{m}\right),
\end{aligned}
$$

where $\varphi_{m}$ is the maximal deviation angle of the droplet axes from the stretching axis, $\operatorname{sinc}(x)=\sin (x) / x$.

\subsection{Transmittance and polarization ability as functions of the angular aperture of the}

\section{optical system}

To analyze the transmittance and polarizing ability of the PDLC layer taking into account the field of view $\theta_{\text {fov }}$ (FOV) angle, it is necessary to integrate the intensity of scattered light over the polar $\theta_{s}$ and azimuthal $\varphi_{s}$ scattering angles from 0 to $\theta_{f o v} / 2$ and from 0 to $2 \pi$, respectively.

Under normal illumination by unpolarized light, the coefficient of incoherent (diffuse) transmission $T_{n p}^{i n c}\left(\theta_{f o v}\right)$ and the integral polarizing ability $P_{\text {inc }}\left(\theta_{f o v}\right)$ of layer for the incoherently scattered light can be determined by:

$$
\begin{gathered}
T_{n p}^{i n c}\left(\theta_{f o v}\right)=\frac{1}{2}\left(T_{\|}^{i n c}\left(\theta_{f o v}\right)+T_{\perp}^{i n c}\left(\theta_{f o v}\right)\right) \\
P_{i n c}\left(\theta_{f o v}\right)=\frac{T_{\perp}^{i n c}\left(\theta_{f o v}\right)-T_{\|}^{i n c}\left(\theta_{f o v}\right)}{T_{\|}^{i n c}\left(\theta_{f o v}\right)+T_{\perp}^{i n c}\left(\theta_{f o v}\right)} \\
T_{\|, \perp}^{i n c}\left(\theta_{f o v}\right)=\frac{1}{C_{n p}} \int_{0}^{2 \pi} d \varphi_{s} \int_{0}^{\theta_{f o v} / 2}\left(I_{v v}^{i n c}\left(\theta_{s}, \varphi_{s}\right)+I_{v h}^{i n c}\left(\theta_{s}, \varphi_{s}\right)\right)_{\alpha=0, \pi / 2} \sin \theta_{s} d \theta_{s} \\
C_{n p}=\int_{0}^{2 \pi} d \varphi_{s} \int_{0}^{\pi}\left\{\left.\left(I_{v v}^{i n c}\left(\theta_{s}, \varphi_{s}\right)+I_{v h}^{i n c}\left(\theta_{s}, \varphi_{s}\right)\right)\right|_{\alpha=0}+\left.\left(I_{v v}^{i n c}\left(\theta_{s}, \varphi_{s}\right)+I_{v h}^{i n c}\left(\theta_{s}, \varphi_{s}\right)\right)\right|_{\alpha=\pi / 2}\right\} \sin \theta_{s} d \theta_{s},
\end{gathered}
$$

where $T_{\|}^{\text {inc }}\left(\theta_{\text {fov }}\right)$ and $T_{\perp}{ }_{\perp}^{\text {inc }}\left(\theta_{\text {fov }}\right)$ are the parallel and orthogonal components of the incoherent transmittance of the layer.

Taking into account the coherent component, the total transmittance $T_{n p}$ of the layer and the polarization degree $P_{f o v}$ are follows:

$$
\begin{aligned}
& T_{n p}\left(\theta_{f o v}\right)=T_{n p}^{c}+T_{n p}^{i n c}\left(\theta_{f o v}\right)=T_{\|}\left(\theta_{f o v}\right)+T_{\perp}\left(\theta_{f o v}\right), \\
& P_{f o v}\left(\theta_{f o v}\right)=\frac{T_{\perp}\left(\theta_{f o v}\right)-T_{\|}\left(\theta_{f o v}\right)}{T_{\|}\left(\theta_{f o v}\right)+T_{\perp}\left(\theta_{f o v}\right)} \\
& T_{\|, \perp}\left(\theta_{f o v}\right)=\frac{1}{2}\left(T_{\|, \perp}^{c}+T_{\|, \perp}^{i n c}\left(\theta_{f o v}\right)\right) .
\end{aligned}
$$

The normalizing factor $C_{n p}$ is determined as: 


$$
\begin{aligned}
& C_{n p}= C \frac{3}{4} \frac{c_{v} l\left\langle a^{2}\right\rangle}{\varepsilon_{y} \varepsilon_{z}\left\langle a^{3}\right\rangle}\left(\left\langle Q_{\|}\right\rangle+\left\langle Q_{\perp}\right\rangle\right), \\
&\left\langle Q_{\|, \perp}\right\rangle=\left(\left\langle Q_{e}\right\rangle \frac{1 \pm S_{2 f}}{2}+\left\langle Q_{e}\right\rangle \frac{1 \mp S_{2 f}}{2}\right), \\
&\left\langle Q_{\|}\right\rangle+\left\langle Q_{\perp}\right\rangle \equiv\left\langle Q_{e}\right\rangle+\left\langle Q_{o}\right\rangle,
\end{aligned}
$$

where $\left\langle Q_{\|}\right\rangle$and $\left\langle Q_{\perp}\right\rangle$ are the average values of extinction efficiency factor of LC droplets for the incident light polarizations parallel and orthogonal to the stretching axis; $\left\langle Q_{e}\right\rangle$ and $\left\langle Q_{o}\right\rangle$ are determined by Eq. (7).

\section{Simulation results}

Here the results of numerical simulation of the polarization degree and the transmittance are presented. They have been obtained by the formulas from the previous section.

The relaxation method of minimizing the free energy volume density was applied to simulate the LC director configuration (a distribution of local optical axes) inside droplets. The anomalous diffraction approximation was used to determine the elements of the amplitude scattering matrix $S_{j}\left(\theta_{s}, \varphi_{s}\right)(j=1,2,3,4)[14,19,22,26-28]$. The calculations were carried out for the monodisperse LC droplets in a polymer film (matrix).

The dependences of the ordinary $n_{\perp}$ and extraordinary $n_{\|}$refractive indices of LC on the wavelength $\lambda$ of incident light were described by Cauchy formulas:

$$
n_{\perp, \|}=A_{\perp, \|}+\frac{B_{\perp, \|}}{\lambda^{2}}+\frac{C_{\perp, \|}}{\lambda^{4}}
$$

where $A_{\perp, \|}, B_{\perp, \|}, C_{\perp, \|}$ are the coefficients determined by the kind of LC used [29].

\subsection{Spectral dependences of the coherent transmittance and the polarization degree of the forward-transmitted light for the stretched PDLC film}

Figure 3 illustrates the simulation data of spectral dependences of the coherent transmittance $T_{n p}^{c}(\lambda)$ and the polarizing ability $P_{c}(\lambda)$ of PDLC film for the forward-transmitted light at two stretching factors of the film $(p=1.16,1.41)$. PDLC films with bipolar, radial and monodomain $y$ configurations of LC inside the droplets are considered. The refractive index $n_{p}$ of polymer matrix was chosen equaled to the ordinary refractive index $n_{\perp}$ of LC at $\lambda=0.62 \mu \mathrm{m} \quad\left(n_{p}=n_{\perp}=1.533\right)$; the extraordinary refractive index is $n_{\|}=1.708$; the layer thickness before stretching was $l_{0}=45 \mu \mathrm{m}$. The calculations were carried out for the monodisperse droplets totally oriented in the stretching 
direction: the variation coefficient [22] of droplet semiaxes $a$ is $D_{a} /\langle a\rangle=0$; the order parameter of film is $S_{2 f}=1$. The rest parameters are shown in the legends of figures. The selected sizes of droplets semiaxis $a=0.93,0.84,0.71 \mu \mathrm{m}$ correspond to the values at which the $T_{n p}^{c}$ and $P_{c}$ are close to the limiting ones: $T_{n p}^{c}(\lambda) \rightarrow 0.5, P_{c}(\lambda) \rightarrow \pm 1.0$, in the wide wavelength range.
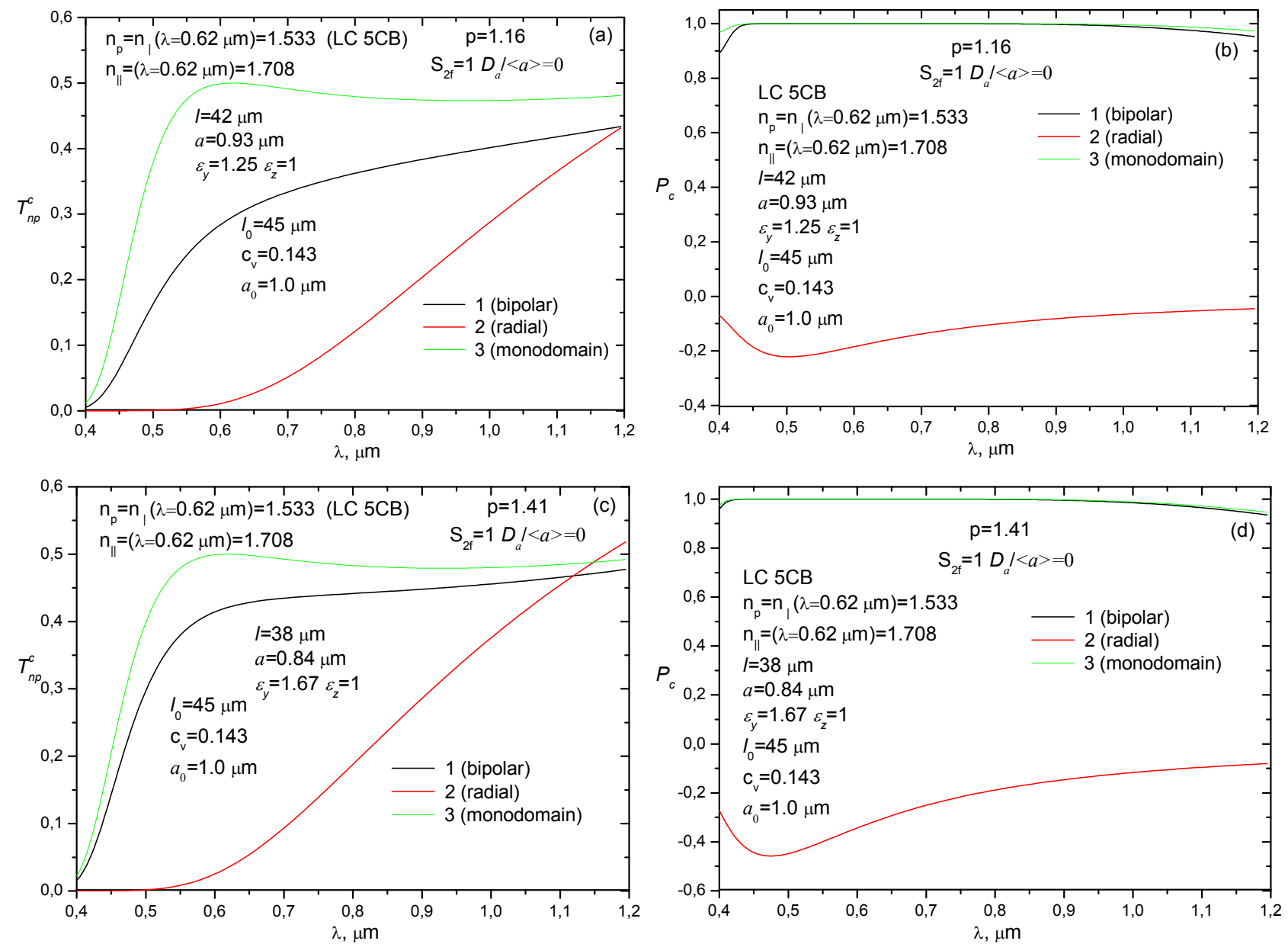

Figure 3. The spectral dependences of the coherent transmittance $T_{n p}^{c}(\lambda)(\mathrm{a}, \mathrm{c})$ and polarization degree $P_{c}(\lambda)$ of the forward-transmitted light $(\mathrm{b}, \mathrm{d})$ at the different stretching factor $p$ of the PDLC films containing droplets with the bipolar (curves 1), radial (curves 2) and monodomain (curves 3) $y$-configuration of LC director. $n_{p}=n_{\perp}=1.533$ at $\lambda=0.62 \mu \mathrm{m}$.

The stretching of PDLC films containing the droplets with monodomain and bipolar configurations allows one to polarize effectively the light in the wavelength range from 0.55 to $0.8 \mu \mathrm{m}$ (polarization degree $P_{c}>0.99$ and transmittance $T_{n p}^{c}>0.47$ ). As calculations show, varying the refractive index of polymer $n_{p}$ (upward at the condition $n_{p}=n_{\perp}(\lambda)$ or $n_{p}=n_{\|}(\lambda)$ ) and the $a$ droplet size, it is possible to shift into the range of smaller wavelength $\lambda<0.55 \mu \mathrm{m}$ with $T_{n p}{ }^{c}(\lambda) \approx$ 0.5 and $P_{c}(\lambda) \approx \pm 1.0$. 
The high polarization degree of forward-transmitted light $\left(P_{c}>0.97\right)$ in the spectral range from 0.45 to $0.7 \mu \mathrm{m}$ (Fig. $3(\mathrm{~b}, \mathrm{~d})$ ) can be achieved in the PDLC films containing LC droplets with the monodomain and bipolar configurations at $n_{p}=n_{\perp}$. The $T_{n p}^{c}$ transmittances of films with bipolar configuration are smaller than that of films with the monodomain internal structure.

The films with the radial droplet structure and the homogeneous surface anchoring do not allow effectively polarize the forward-transmitted light.

Note, the positive and negative values of $P_{c}$ mean that the forward-transmitted light is polarized orthogonally and parallel to the direction of film stretching, respectively. A polarization sign is determined by which component (polarized orthogonally or parallel to the stretching axis) is less scattered. For the monodomain LC structure the orthogonal component of transmittance is not scattered at wavelength $\lambda$, if $n_{p}=n_{\perp}(\lambda)$. At that, if the parallel component is strongly scattered, the polarization degree of the forward-transmitted light $P_{c} \rightarrow+1$ (forward-transmitted light is linearly polarized orthogonally to the stretching axis). For $n_{p}=n_{\|}(\lambda)$ the parallel component is not scattered. And $P_{c} \rightarrow-1$ (forward-transmitted light is linearly polarized parallel to the stretching axis) if the orthogonal component is strongly scattered.

\subsection{Monodomain structure}

It is convenient to consider the transmittance and polarization by varying the $a$-values in the conditions of: (i) the equality of the refractive index $n_{p}$ of polymer to the ordinary refractive index $n_{\perp}$ of LC at the selected wavelength $\lambda\left(n_{p}=n_{\perp}(\lambda)\right)$ or (ii) the equality of $n_{p}$ to the extraordinary refractive index $n_{\|}$of LC $\left(n_{p}=n_{\|}(\lambda)\right)$.

Figures 4 and 5 show the calculation data for 5CB liquid crystal in the red, green and blue spectral ranges $(\lambda=0.62,0.5$ and $0.45 \mu \mathrm{m})$. As can see, if $n_{p}=n_{\perp}(\lambda)$ or $n_{p}=n_{\|}(\lambda)$, the maximum $T_{n p}^{c}$ $\approx 0.5$ is realized at these $\lambda$ for all considered sizes of $a$ semiaxis (Fig. 4(a,c,e) and Fig. 5(a,c,e)). The values of the refractive index $n_{p}$ of polymer and $a$ semiaxis determine the wavelength range where the transmittance $T_{n p}^{c}$ of the film and polarization degree $P_{c}$ of light reach simultaneously the values close to the limiting ones $(0.5$ and \pm 1.0$)$, respectively. For $a=0.7 \mu \mathrm{m}$ the polarization degree of forward-transmitted light has the values close to the maximal one $\left(P_{c} \approx \pm 1\right)$ in the wide enough spectral range (Fig. 4(b,d,f) and Fig. 5(b,d,f)). For the film with the parameters $l=32 \mu \mathrm{m}, c_{v}=0.143$, $\varepsilon_{y}=2.83, \varepsilon_{z}=1.0$ containing the monodisperse oriented droplets, the value $a=0.7 \mu \mathrm{m}$ allows realizing the spectral characteristics close to the limiting ones. 

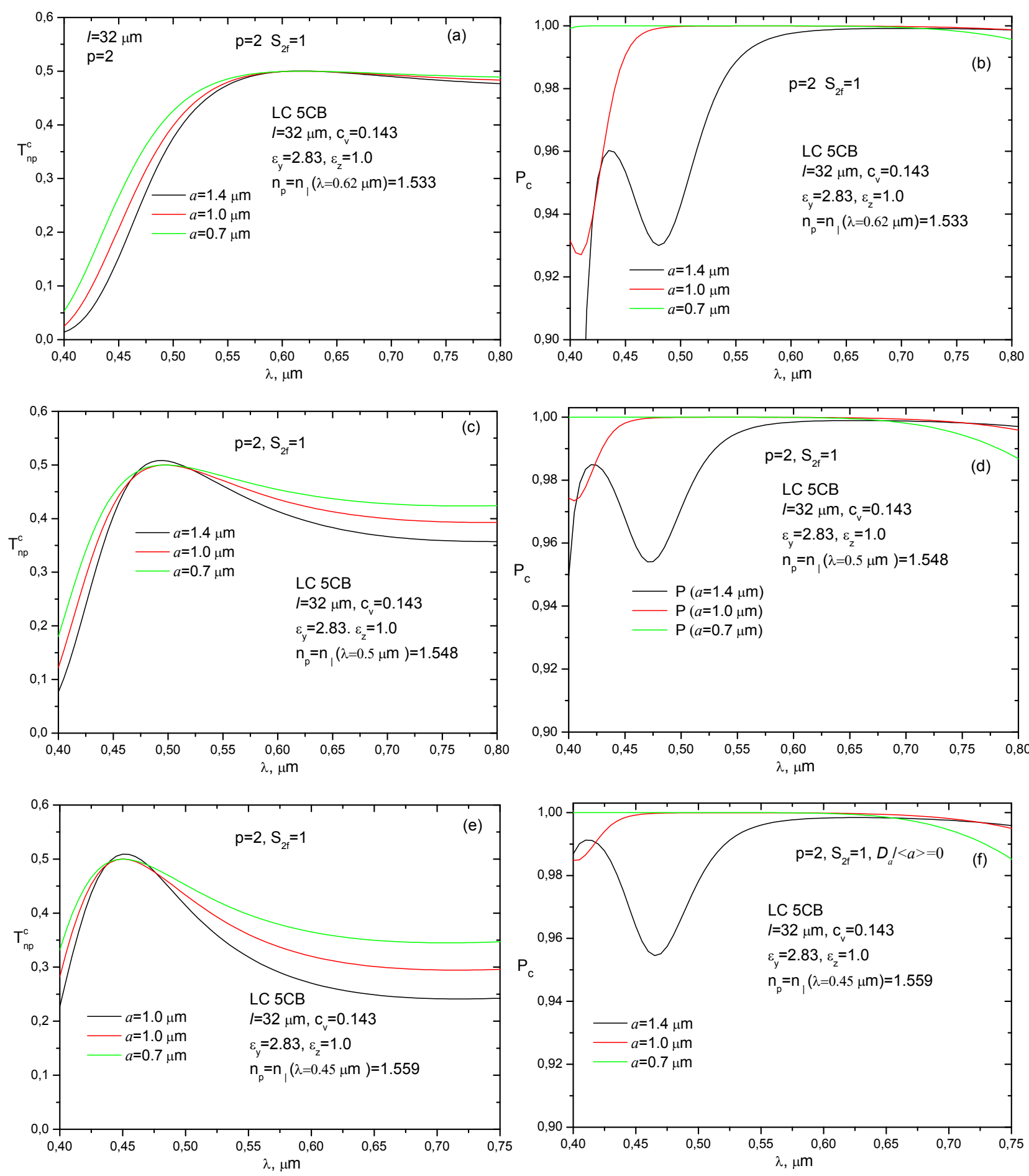

Figure 4. The spectral dependences of coherent transmittance $T_{n p}^{c}(\mathrm{a}, \mathrm{c}, \mathrm{e})$ and polarization degree $P_{c}(\mathrm{~b}, \mathrm{~d}, \mathrm{f})$ of the forward-transmitted light at the different sizes of lateral droplet semiaxis $a$ for the polymer refractive index $n_{p}$ equaled to the ordinary refractive index $n_{\perp}$ of $5 \mathrm{CB}$ at $\lambda=0.62(\mathrm{a}, \mathrm{b}) ; 0.5$ (c, d) and $0.45 \mu \mathrm{m}(\mathrm{e}, \mathrm{f})$. Monodisperse droplets with the monodomain LC structure and oriented optical axes (the film order parameter $S_{2 f}=1$ ). The stretching ratio of film is $p=2$, the droplet lateral semiaxis is $a=0.7 \mu \mathrm{m}$, the film thickness is $l=32 \mu \mathrm{m}$, the volume filling factor of PDLC film is $c_{v}=0.143$, the anisometry parameters of LC droplets are $\varepsilon_{y}=2.83, \varepsilon_{z}=1.0$. 

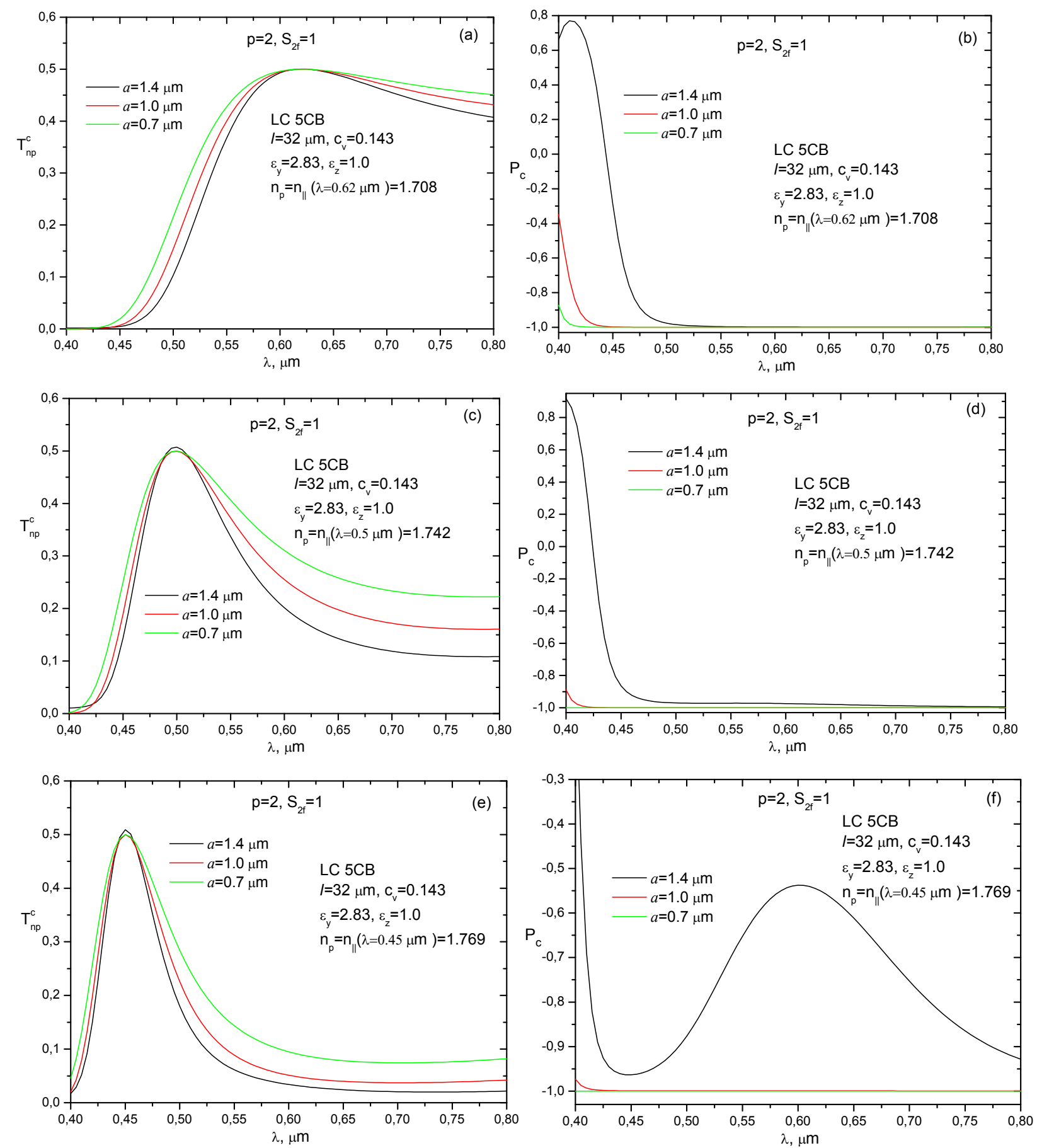

Figure 5. The spectral dependences of coherent transmittance $T_{n p}^{c}(\mathrm{a}, \mathrm{c}, \mathrm{e})$ and polarization degree $P_{c}(\mathrm{~b}, \mathrm{~d}, \mathrm{f})$ of the forward-transmitted light at the different sizes of lateral droplet semiaxis $a$ for the polymer refractive index $n_{p}$ equaled to the extraordinary refractive index $n_{\|}$of $5 \mathrm{CB}$ at $\lambda=0.62(\mathrm{a}, \mathrm{b})$; 0.5 (c, d) and $0.45 \mu \mathrm{m}(\mathrm{e}, \mathrm{f})$. Monodisperse droplets with the monodomain LC structure and oriented optical axes. $S_{2 f}=1, p=2, a=0.7 \mu \mathrm{m}, l=32 \mu \mathrm{m}, c_{v}=0.143, \varepsilon_{y}=2.83, \varepsilon_{z}=1.0$.

Figure 6 illustrates the $T_{n p}^{c}(\lambda)$ and $P_{c}(\lambda)$ dependences for the film with 5CB LC droplets at $n_{p}=n_{\perp, \|}(\lambda=0.62,0.5,0.45 \mu \mathrm{m})$. As can see, an effective polarization of visible light in the 
wavelength range from blue to red with significant coherent transmission coefficient can be achieved by changing the refractive index $n_{p}$ of polymer from $n_{p}=n_{\perp}$ to $n_{p}=n_{\|}$.
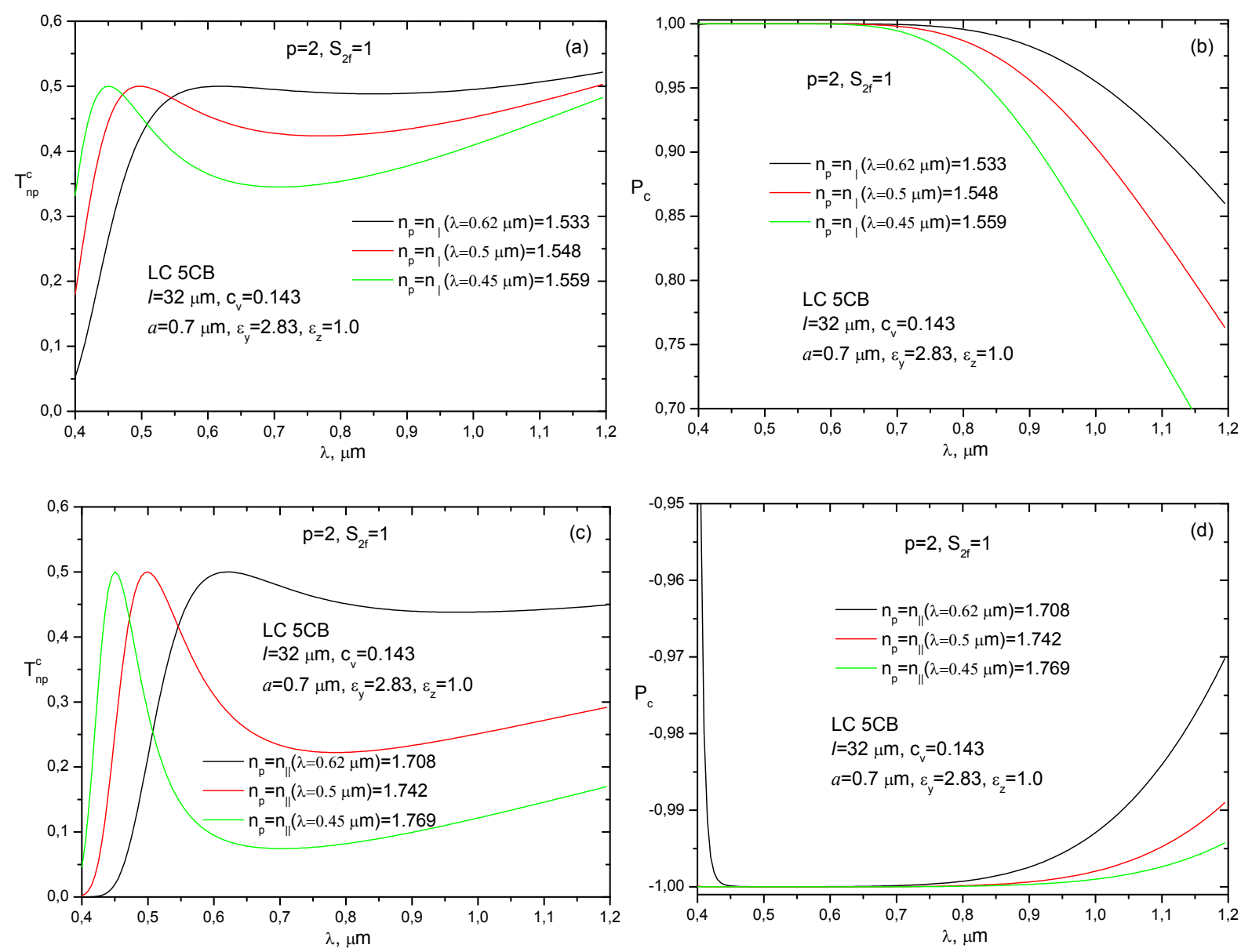

Figure 6. The dependences of coherent transmittance $T_{n p}^{c}$ of PDLC film (a,c) and polarization degree $P_{c}(\mathrm{~b}, \mathrm{~d})$ of the forward-transmitted light on the wavelength $\lambda$ of incident unpolarized light for the polymer refractive indices $n_{p}$ equaled to the ordinary $n_{\perp}(\mathrm{a}, \mathrm{b})$ and extraordinary $n_{\|}(\mathrm{c}, \mathrm{d})$ refractive indices of $5 \mathrm{CB}$ at $\lambda=0.62,0.5$ and $0.45 \mu \mathrm{m}$. Monodisperse droplets with the monodomain LC structure and oriented optical axes (the film order parameter $S_{2 f}=1$ ). The stretching ratio of film is $p=2$, the droplet lateral semiaxis is $a=0.7 \mu \mathrm{m}$, the film thickness is $l=32 \mu \mathrm{m}$, the volume filling factor of PDLC film is $c_{v}=0.143$, the anisometry parameters of LC droplets are $\varepsilon_{y}=b / a=2.83$, $\varepsilon_{z}=c / a=1.0$ (where $b$ is the droplet semiaxis along the stretching direction, $a$ is the droplet semiaxis along the normal to the film, $c$ is the lateral semiaxis in the film plane).

The $T_{n p}^{c}(\lambda)$ and $P_{c}(\lambda)$ dependences for PDLC films based on the different liquid crystals (5CB, E7, E44) are shown in Figure 7. An increasing of the optical anisotropy of LC allows widening the wavelength range in which the polarizing ability of the film will be $P \approx \pm 1.0$ (Fig. 7(b), $7(d))$. 

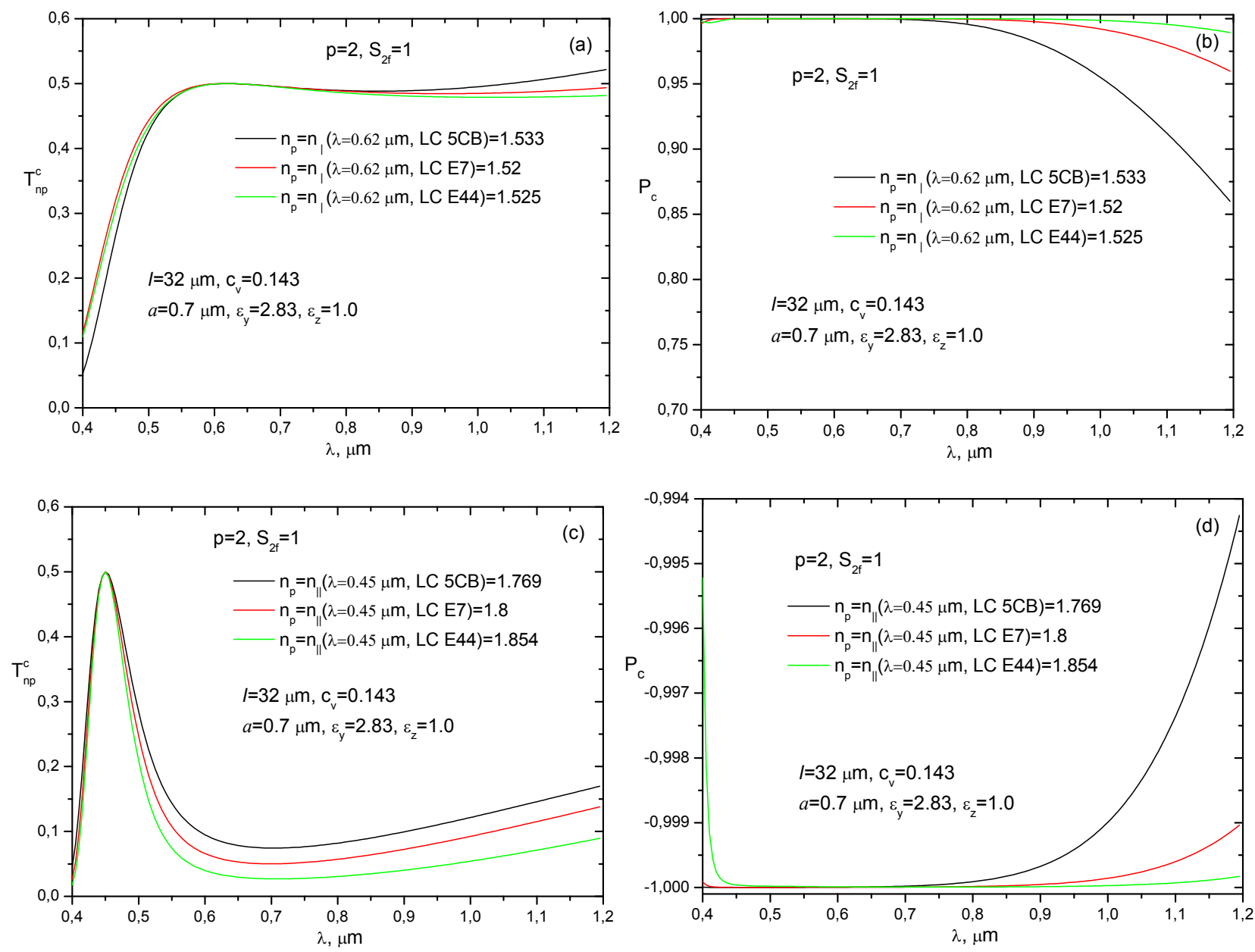

Figure 7. The dependences of coherent transmittance $T_{n p}^{c}$ of PDLC film (a,c) and polarization degree $P_{c}(\mathrm{~b}, \mathrm{~d})$ of the forward-transmitted light on the wavelength $\lambda$ of incident unpolarized light for the polymer refractive indices $n_{p}$ equaled to the ordinary $n_{\perp}(\mathrm{a}, \mathrm{b})$ and extraordinary $n_{\|}(\mathrm{c}, \mathrm{d})$ refractive indices of different $\operatorname{LC}(5 \mathrm{CB}, \mathrm{E} 7, \mathrm{E} 44)$ at $\lambda=0.62 \mu \mathrm{m}(\mathrm{a}, \mathrm{b})$ and $\lambda=0.45 \mu \mathrm{m}(\mathrm{c}, \mathrm{d})$. Monodisperse droplets with the monodomain LC structure and oriented optical axes $\left(S_{2 f}=1\right)$. $p=2$, $a=0.7 \mu \mathrm{m}, l=32 \mu \mathrm{m}, c_{v}=0.143, \varepsilon_{y}=2.83, \varepsilon_{z}=1.0$.

The results shown in Figures 6 and 7 imply that the mechanically stretched PDLC films in which the droplet-polymer interface is modified by surfactants can be used as: (i) the wide-band polarizers of forward-transmitted light with the high transmittance $\left(T_{n p}^{c} \approx 0.5\right)$ and the polarizing ability $\left(P_{c}(\lambda) \approx \pm 1\right)$ in the wavelength range $0.6 \mu \mathrm{m}<\lambda<0.7 \mu \mathrm{m}$; (ii) the polarizers with the transmittance $T_{n p}^{c} \approx 0.5$ and the polarization degree $P_{c} \approx-1.0$ at $\lambda \approx 0.5 \mu \mathrm{m}$ (Fig. 7(c), 7(d)), the polarizers at $\lambda \approx 0.45 \mu \mathrm{m}$ and at others wavelengths of visible light. The values $P_{c}=1$ and $P_{c}=1$ in the Figures 4-7 mean that the forward-transmitted light is linearly polarized orthogonally and parallel to the film stretching direction, respectively. As was mentioned above, this is determined by which of the transmittance components is not scattered. 
The near to limiting values $T_{n p}^{c} \approx 0.5$ and $P_{c} \approx 1.0$ (or $P_{c} \approx-1$ ) depend on a droplet polydispersity as well as orientation of their optical axes under film stretching (Fig. 8). Increasing the droplet polydispersity (described by the variation coefficient $D_{a} /\langle a\rangle$ ) and decreasing the order parameter $S_{2 f}$ of film result in reduce of its coherent transmittance $T_{n p}^{c}$ (Fig. 8(a, c, e)) and change of the spectral dependence of the polarization degree $P_{c}$ of forward-transmitted light (Fig. 8(b, d, f)).
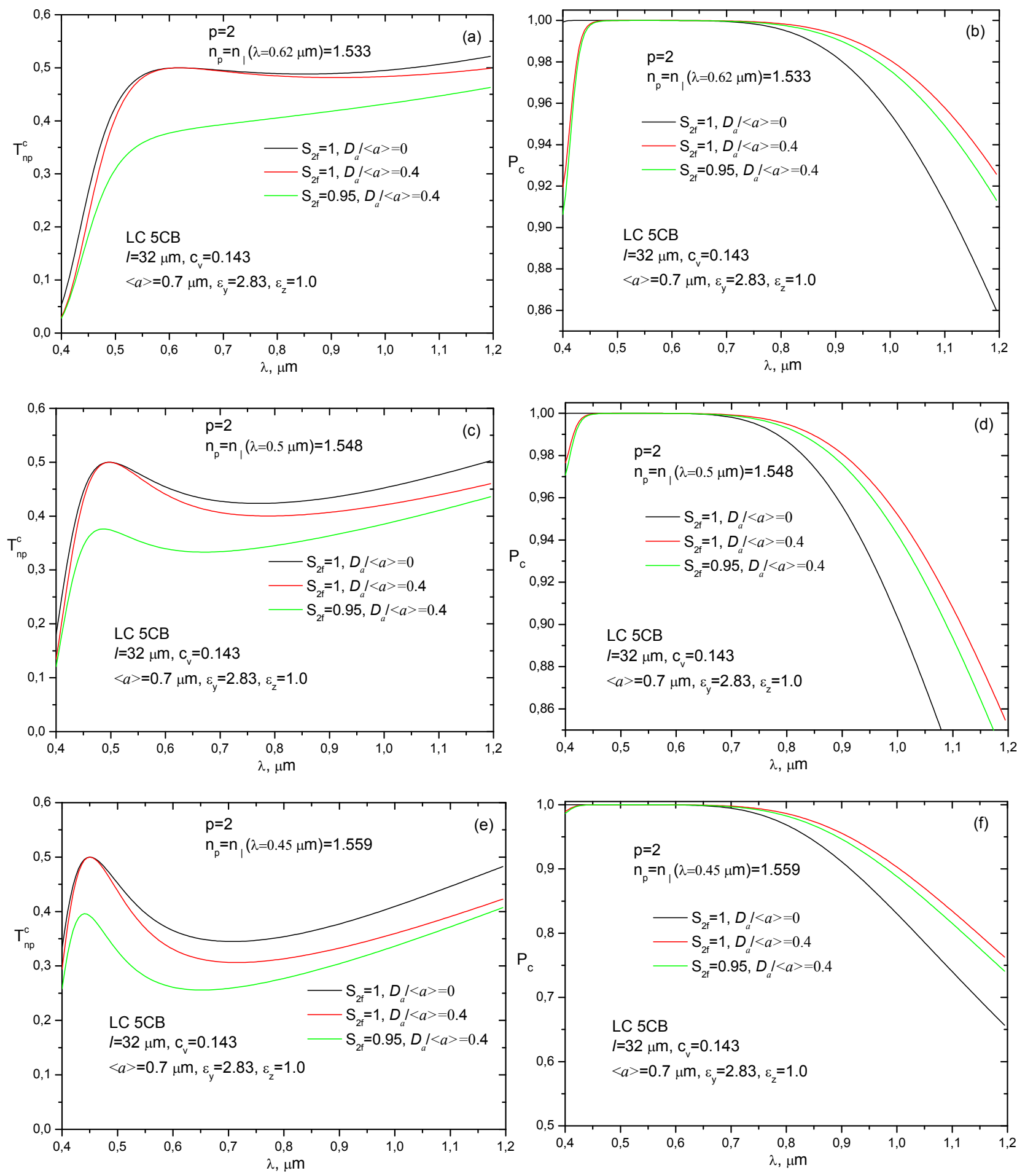

Figure 8. The spectral dependences of coherent transmittance $T_{n p}^{c}$ of PDLC film (a,c,e) illuminated by unpolarized incident light and the polarization degree $P_{c}(\mathrm{~b}, \mathrm{~d}, \mathrm{f})$ of the forward- 
transmitted light in condition of equality of the polymer refractive index $n_{p}$ and the ordinary refractive index $n_{\perp}$ of $5 \mathrm{CB}$ at $\lambda=0.62,0.5$ and $0.45 \mu \mathrm{m}$. The LC structure inside the droplets is monodomain. The stretching ratio of film is $p=2$. The average size of droplet lateral semiaxis $a$ is $\langle a\rangle=0.7 \mu \mathrm{m}$. The anisometry parameters of droplets are $\varepsilon_{y}=2.83$, and $\varepsilon_{z}=1.0$. The order parameters of film are $S_{2 f}=1$ and 0.95 . Gamma-distribution of droplet sizes. The variation coefficients are $D_{a} /\langle a\rangle=0$ and 0.4 .

The numerical analysis showed that the narrowing of spectral range in which the film transmittance is close to the limiting values $\left(T_{n p}^{c} \approx 0.5\right)$ is most sensitive to a variation of film order parameter $S_{2 f}$ (i.e. to the difference of $S_{2 f}$ values from 1 corresponding to a perfect orientation of droplet optical axes under mechanical stretching). The polarization degree $P_{c}$ of the forwardtransmitted light is most sensitive to a polydispersity of LC droplets.

\subsection{Small-angle distribution of the intensity and polarization degree of the scattered}

\section{light. Droplets with homogeneous and inhomogeneous surface anchoring}

Figures 9, 10 illustrate the dependences of the $I_{\|}^{i n c_{-}}, I_{\perp}{ }^{i n c_{-}}$-components of scattered light, and the polarization degree $P_{\text {inc }}$ of scattered light on the polar $\theta_{s}$ and azimuthal $\varphi_{s}$ scattering angles. The simulation was carried out at double stretching of PDLC films $(p=2)$ with the homogeneous surface anchoring for the bipolar and radial LC configurations inside the droplets and with the inhomogeneous anchoring for the monodomain structure. The refractive index of polymer $n_{p}=n_{\perp}=1.533$ at $\lambda=0.62 \mu \mathrm{m}$. Other parameters of the simulations are indicated in the legends of figures. 

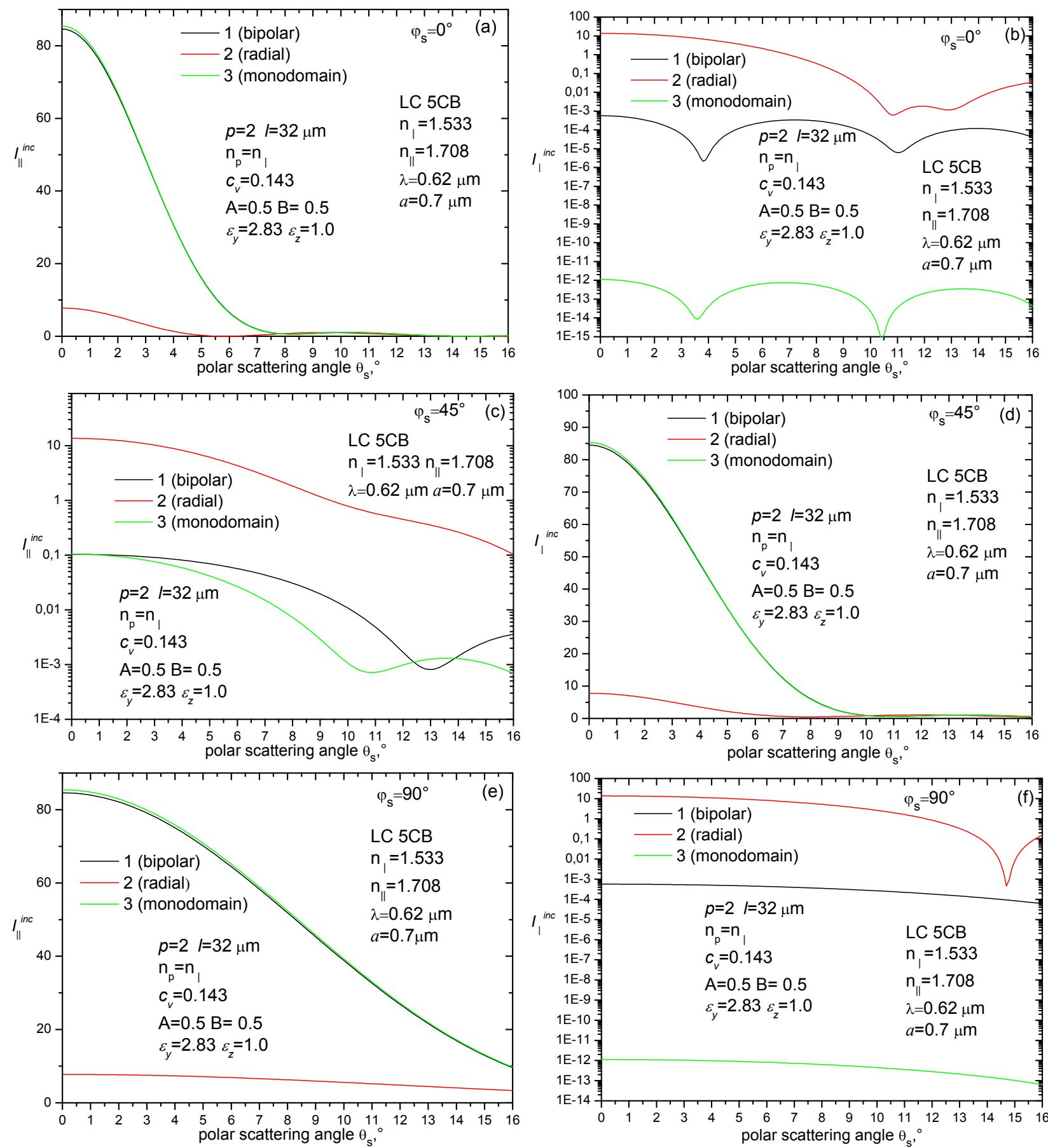

Figure 9. The dependences of parallel (to the stretching axis) $I_{\|}{ }^{i n c}\left(\theta_{S}\right)$ (a, c, e) and orthogonal $I_{\perp}{ }^{i n c}\left(\theta_{s}\right)(\mathrm{b}, \mathrm{d}, \mathrm{f})$ components of scattered light intensity on the polar scattering angle $\theta_{s}$ at different azimuthal scattering angles $\varphi_{s}$. PDLC film was double-stretched $(p=2)$ at homogeneous $(1,2)$ and inhomogeneous (3) surface anchoring. $n_{p}=n_{\perp}=1.533$ at $\lambda=0.62 \mu \mathrm{m}$.

As can see in Figures $9(\mathrm{a}, \mathrm{b})$ and $9(\mathrm{e}, \mathrm{f})$, at $\varphi_{s}=0^{\circ}, 90^{\circ}$ a high anisotropy of light scattering is observed for both the bipolar and monodomain droplet structures: the ratios $I_{\|}^{\text {inc }}\left(\theta_{S}\right) / I_{\perp}{ }_{\perp}$ inc $\left(\theta_{s}\right) \approx 10^{3}$ for bipolar structure and $I_{\|}^{i n c}\left(\theta_{s}\right) / I_{\perp}^{i n c}\left(\theta_{s}\right) \approx 10^{12}$ for monodomain configuration. At $\varphi_{s}=45^{\circ}$ (Fig. 9 (c, d)), $I_{\|}^{\text {inc }}\left(\theta_{s}\right) / I_{\perp}{ }^{i n c}\left(\theta_{s}\right) \approx 10^{-4}$ both for the bipolar and monodomain droplet structures. For the PDLC films 
containing the droplets with radial structure, the ratios $I_{\|}^{i n c}\left(\theta_{s}\right) / I_{\perp}{ }^{i n c}\left(\theta_{s}\right) \approx 1 / 2$ at $\varphi_{s}=0^{\circ} ; 90^{\circ}$ and $I_{\|}{ }^{\text {inc }}\left(\theta_{S}\right) / I_{\perp}{ }^{\text {inc }}\left(\theta_{S}\right) \approx 2$ at $\varphi_{s}=45^{\circ}$.

The intensity $I_{n p}^{i n c}\left(\theta_{s}, \varphi_{s}\right)$ (Fig. $\left.10(\mathrm{a}, \mathrm{c}, \mathrm{e})\right)$ and the polarization degree $P_{\text {inc }}$ of incoherently scattered light (Fig. $10(\mathrm{~b}, \mathrm{~d}, \mathrm{f}))$ practically coincide with $I_{n p}^{i n c}\left(\theta_{s}, \varphi_{s}\right)$ and $P_{\text {inc }}$, respectively, for the double-stretched PDLC films with bipolar and monodomain structures.
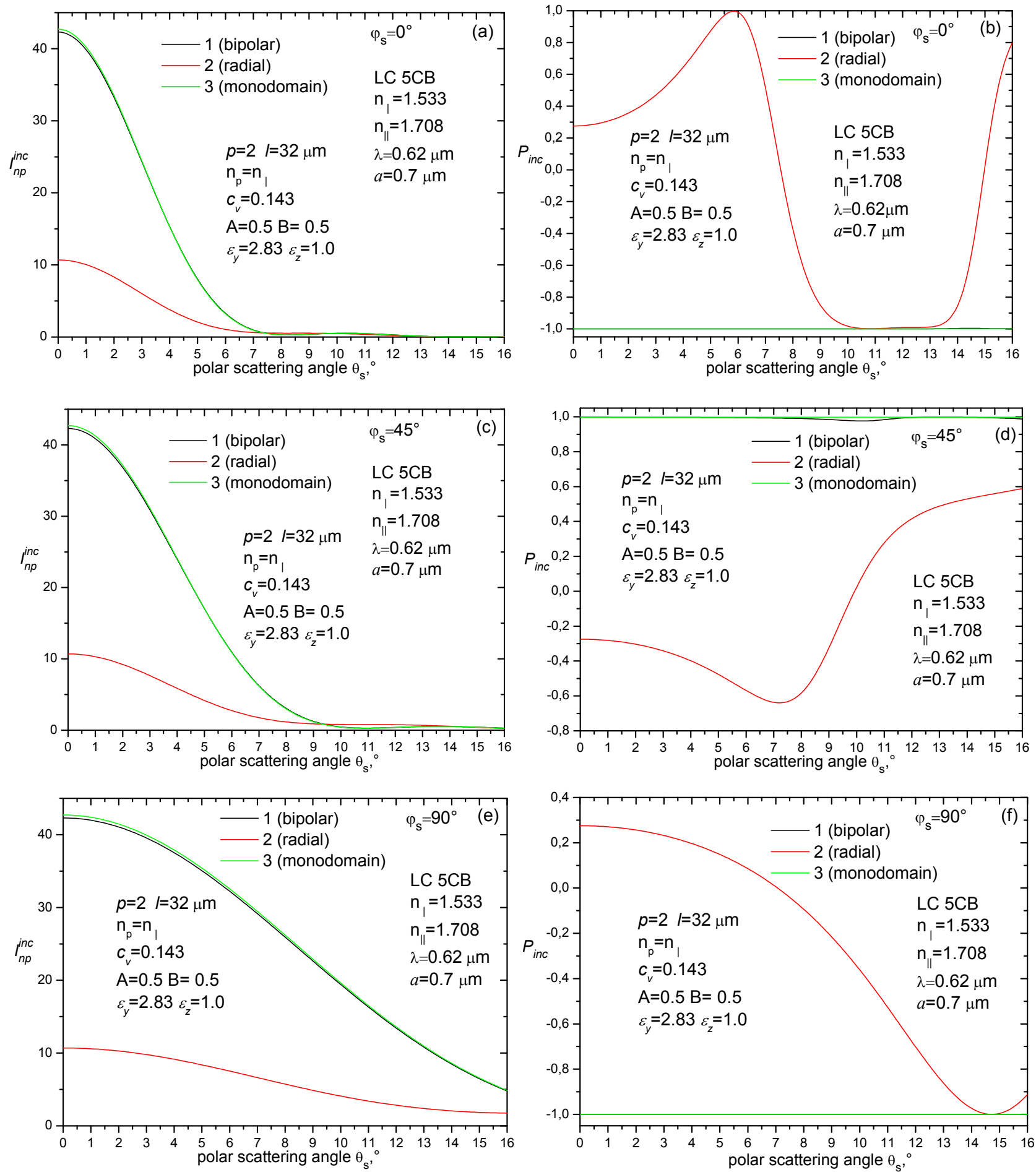

Figure 10. The dependences of intensity $I_{n p}^{\text {inc }}\left(\theta_{s}\right)(\mathrm{a}, \mathrm{c}, \mathrm{e})$ and polarization degree $P_{\text {inc }}\left(\theta_{s}\right)(\mathrm{b}, \mathrm{d}, \mathrm{f})$ of scattered light on the polar scattering angle $\theta_{s}$ at different values of azimuthal scattering angle $\varphi_{s}$. 
PDLC film was double-stretched $(p=2)$ at homogeneous $(1,2)$ and inhomogeneous (3) surface anchoring. $n_{p}=n_{\perp}=1.533$ at $\lambda=0.62 \mu \mathrm{m}$.

The polarization degree $P_{i n c}$ of incoherently scattered light does not practically depend on the polar scattering angle $\theta_{s}$ (Fig. $10(\mathrm{~b}, \mathrm{~d}, \mathrm{f})$ ) for bipolar and monodomain structures. It is caused by the equality $n_{p}=n_{\perp}$ when one of the components (parallel, $I_{\|}^{\text {inc }}\left(\theta_{s}\right)$, or orthogonal, $I_{\perp}{ }_{\perp}$ inc $\left(\theta_{s}\right)$ ) exceeds significantly another one (by 4-12 orders and more, Fig. 9). At $I_{\|}^{\text {inc }}\left(\theta_{s}\right) / I_{\perp}{ }^{\text {inc }}\left(\theta_{s}\right) \rightarrow \infty$, the value $P_{\text {inc }}\left(\theta_{s}\right) \rightarrow-1$. At $I_{\|} \|^{i n c}\left(\theta_{s}\right) / I_{\perp}{ }_{\perp}$ inc $\left(\theta_{s}\right) \rightarrow 0$, the value $P_{\text {inc }}\left(\theta_{s}\right) \rightarrow+1$. Figure $10(\mathrm{~b}, \mathrm{~d}, \mathrm{f})$ shows the polarization degree $P_{i n c}=-1$ at $\varphi_{s}=0^{\circ}, 90^{\circ}$ and $P_{i n c}=+1$ for $\varphi_{S}=45^{\circ}-$ for bipolar and monodomain structures. It should be emphasized that the polarization degree $P_{\text {inc }}\left(\theta_{s}\right)$ of diffusely scattered light for stretched PDLC film containing radial droplets (Fig. $10(\mathrm{~b}, \mathrm{f}))$ can reach the limiting values $( \pm 1)$ at the azimuthal $\varphi_{s}=0^{\circ} ; 90^{\circ}$ and polar $\theta_{s} \approx 6^{\circ} ; 12^{\circ} ; 15^{\circ}$ etc. scattering angles.

\subsection{Small-angle distribution of the intensity and polarization degree of the scattered} light. Droplets with monodomain structure: refractive index of polymer is equal to the ordinary refractive index of liquid crystal

Figures 11, 12 display the parallel $I_{\|}^{i n c}$ and orthogonal $I_{\perp}^{i n c}$ (to the stretching axis) components of scattered light intensity, the intensity $I_{n p}^{i n c}$ of scattered light for unpolarized incident light and the polarization degree $P_{i n c}$ of incoherently scattered light. The calculations were performed for a double-stretched PDLC film with monodomain droplet structure forming under modification of surface anchoring for initially (before stretching) spherical droplets with bipolar and radial configurations. The refractive index $n_{p}$ of polymer matrix is equal to the ordinary refractive index $n_{\perp}$ of $5 \mathrm{CB}$ at $\lambda=0.62,0.5,0.45 \mu \mathrm{m}$. The following parameters were used in the simulation: the film thickness is $l=32 \mu \mathrm{m}$; the stretching ratio is $p=2$ (the film thickness before stretching was $l_{0}=45 \mu \mathrm{m}$ ); the volume filling factor of film is $c_{v}=0.143$; the anisometry parameters of droplets are $\varepsilon_{y}=2.83, \varepsilon_{z}=1.0(p=2)$; the droplet semiaxis $a=0.7 \mu \mathrm{m}$. 

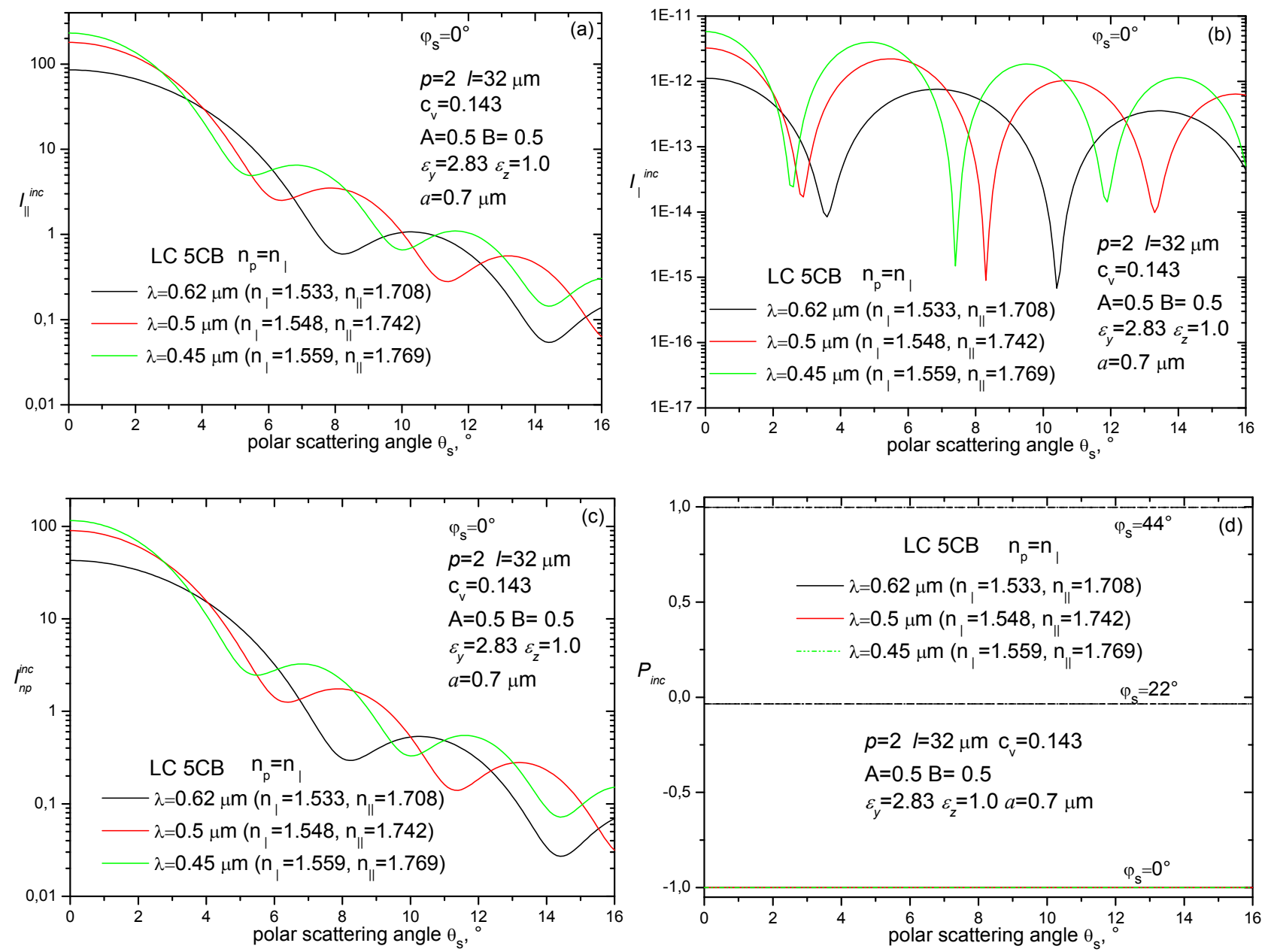

Figure 11. Angular distribution of the parallel $I_{\|}^{\text {inc }}\left(\theta_{s}\right)$ (a) and orthogonal $I_{\perp}{ }^{\text {inc }}\left(\theta_{s}\right)$ (b) (to the stretching axis) intensity components, the intensity $I_{n p}^{i n c}\left(\theta_{s}\right)(\mathrm{c})$, and the polarization degree $P_{\text {inc }}\left(\theta_{s}\right)$ (d) of scattered light for the refractive index $n_{p}$ of polymer matrix equaled to the ordinary refractive index $n_{\perp}$ of $5 \mathrm{CB}$ at $\lambda=0.62,0.5,0.45 \mu \mathrm{m}$. The stretching ratio of film is $p=2$. The structure within LC droplets is monodomain. $a=0.7 \mu \mathrm{m}$.

Figure 11 shows that a high asymmetry of scattered light reveals for all considered wavelengths. The ratio $I_{\|}^{i n c}\left(\theta_{S}\right) / I_{\perp}{ }^{i n c}\left(\theta_{S}\right)>10^{12}$ at $\varphi_{S}=0^{\circ}$ (Fig. $\left.11(\mathrm{a}, \mathrm{b})\right)$.

It is worth noting, that at the considered conditions $\left(n_{p}=n_{\perp}\right)$, the polarization degree $P_{i n c}$ is the same at different wavelengths and does not depend on the polar scattering angle $\theta_{s}$. At that $P_{\text {inc }}$ depends on the azimuthal angle (Fig. 11 (d), Fig. 12). 


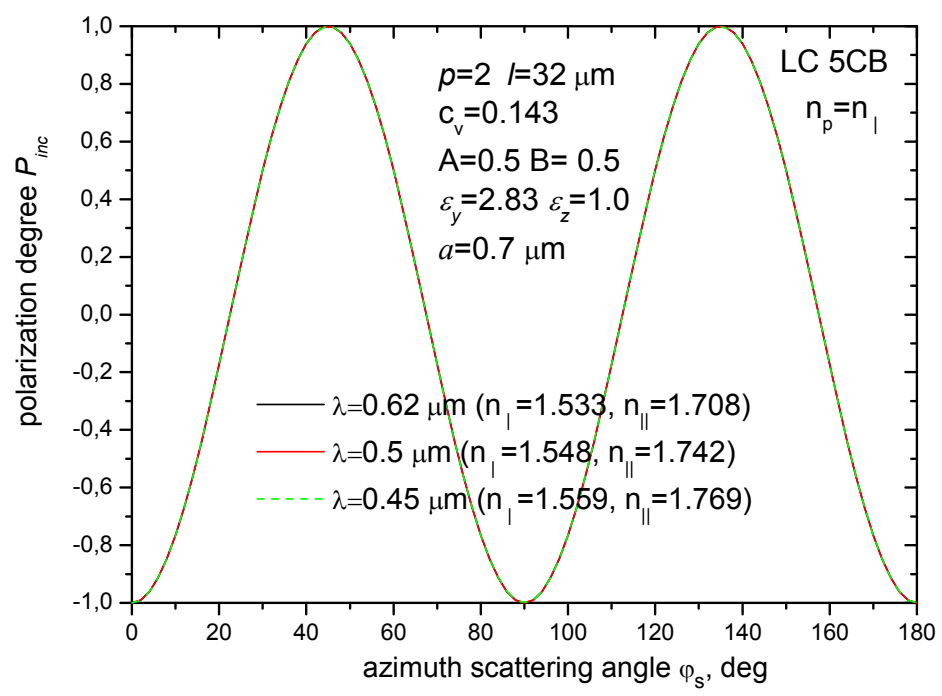

Figure 12. Dependences of the polarization degree $P_{\text {inc }}$ of scattered light on the azimuthal scattering angle $\varphi_{s}$ in the condition of the equality of the refractive index $n_{p}$ of polymer matrix and ordinary refractive index $n_{\perp}$ of $5 \mathrm{CB}$ at $\lambda=0.62,0.5,0.45 \mu \mathrm{m}$. The stretching ratio of film is $p=2$. The structure within LC droplets is monodomain. $a=0.7 \mu \mathrm{m}$.

Figures 13-16 demonstrate the angular distributions of intensity components and the polarization degree of incoherently scattered light for the stretched PDLC film containing monodisperse droplets with monodomain director configuration and with totally oriented structure of their $\mathbf{N}_{j}$ optical axes. The dependences of $I_{v v, v h}^{i n c}\left(\theta_{s}, \varphi_{s}\right), I_{n p}^{i n c}\left(\theta_{s}, \varphi_{s}\right)$ and $P_{i n c}\left(\theta_{s}, \varphi_{s}\right)$ were calculated for $\mathrm{LC} 5 \mathrm{CB}$ according to the formulas of Section 2.2 at the following parameters: $\theta_{f o v}=16^{\circ}, p=2, l=32 \mu \mathrm{m}, c_{v}=0.143, a=1.4 \mu \mathrm{m}, \varepsilon_{y}=2.83, \varepsilon_{z}=1.0, A=B=0.5, C=1$. The horizontal and vertical axes show the values of $y=\sin \theta_{s} \cos \varphi_{s}$ and $z=\sin \theta_{s} \sin \varphi_{s}$ within $-8^{\circ} \leq \theta_{s} \leq 8^{\circ}, 0^{\circ} \leq \varphi_{s} \leq 360^{\circ}$.

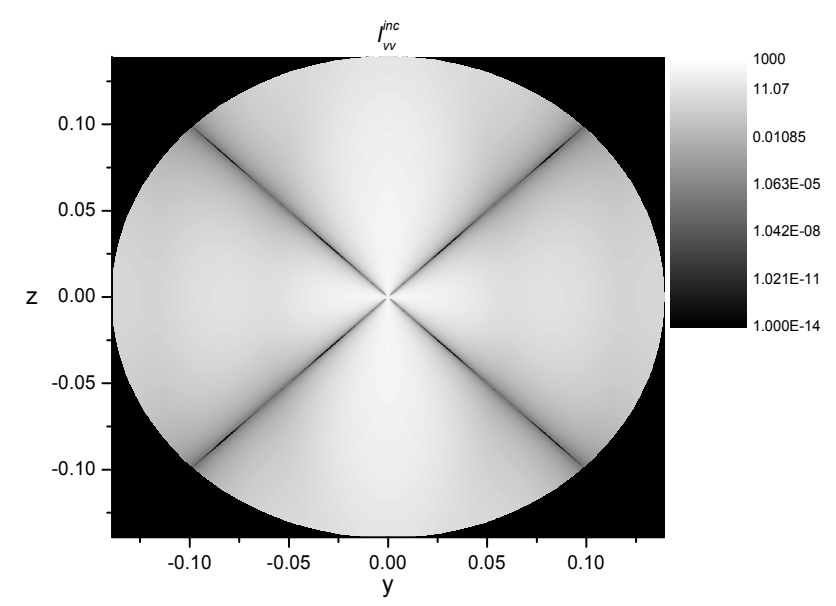

Figure 13. Distribution of the $I_{v v}^{i n c}$-component of incoherently scattered light. 


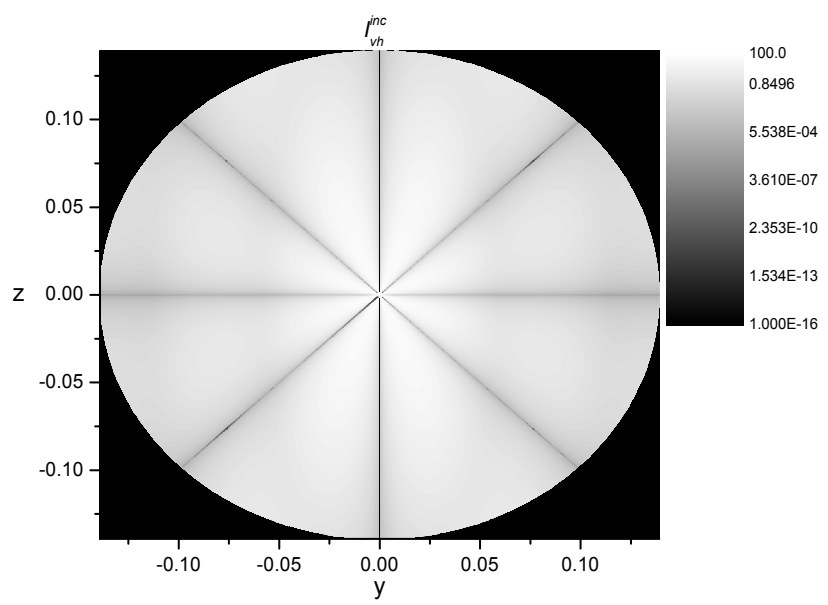

Figure 14. Distribution of the $I_{v h}^{i n c}$-component of incoherently scattered light.

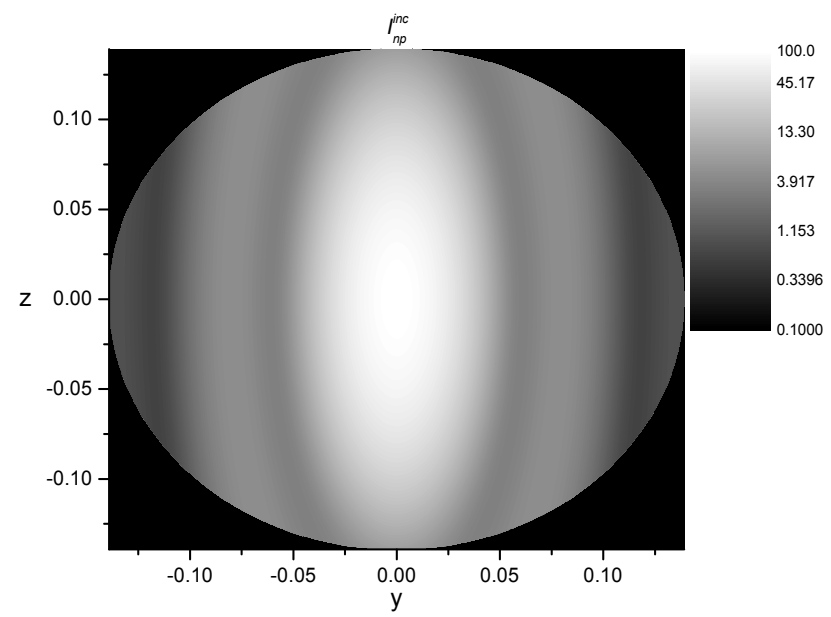

Figure 15. Distribution of the $I_{n p}^{i n c}$ intensity of incoherently scattered light.

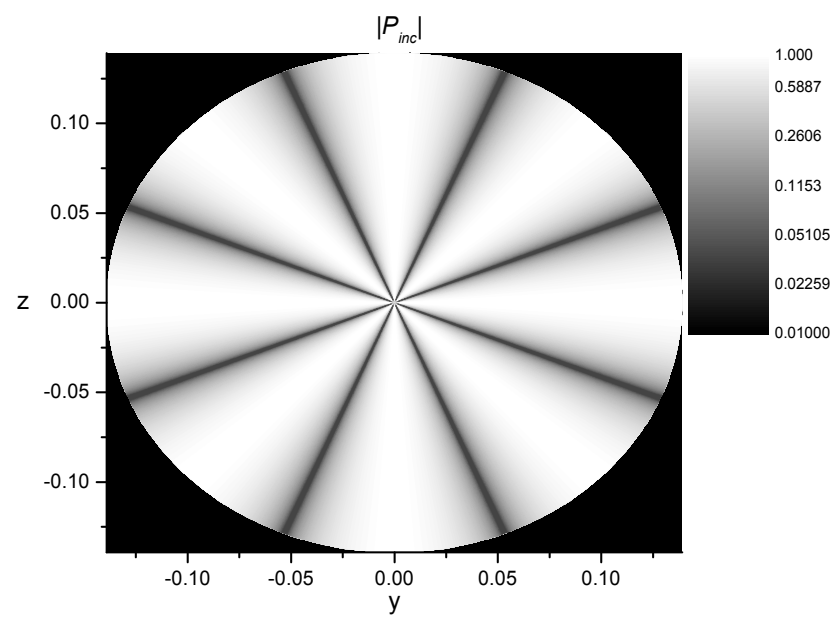

Figure 16. Distribution of the $\left|P_{\text {inc }}\right|$ for incoherently scattered light.

3.5 Dependence of the transmittance and polarizing ability of the film with the monodomain droplet structure on the field of view 
The dependences of the $T_{\|, \perp}^{i n c}, T_{\|, \perp}, T_{n p}^{i n c}, T_{n p}$ transmittances and polarizing abilities $P_{f o v}^{i n c}, P_{f o v}$ of PDLC film on the field of view $\theta_{f o v}$ (angular aperture of the optical system recording the intensity of light scattered by the film) are shown in Figure 17.
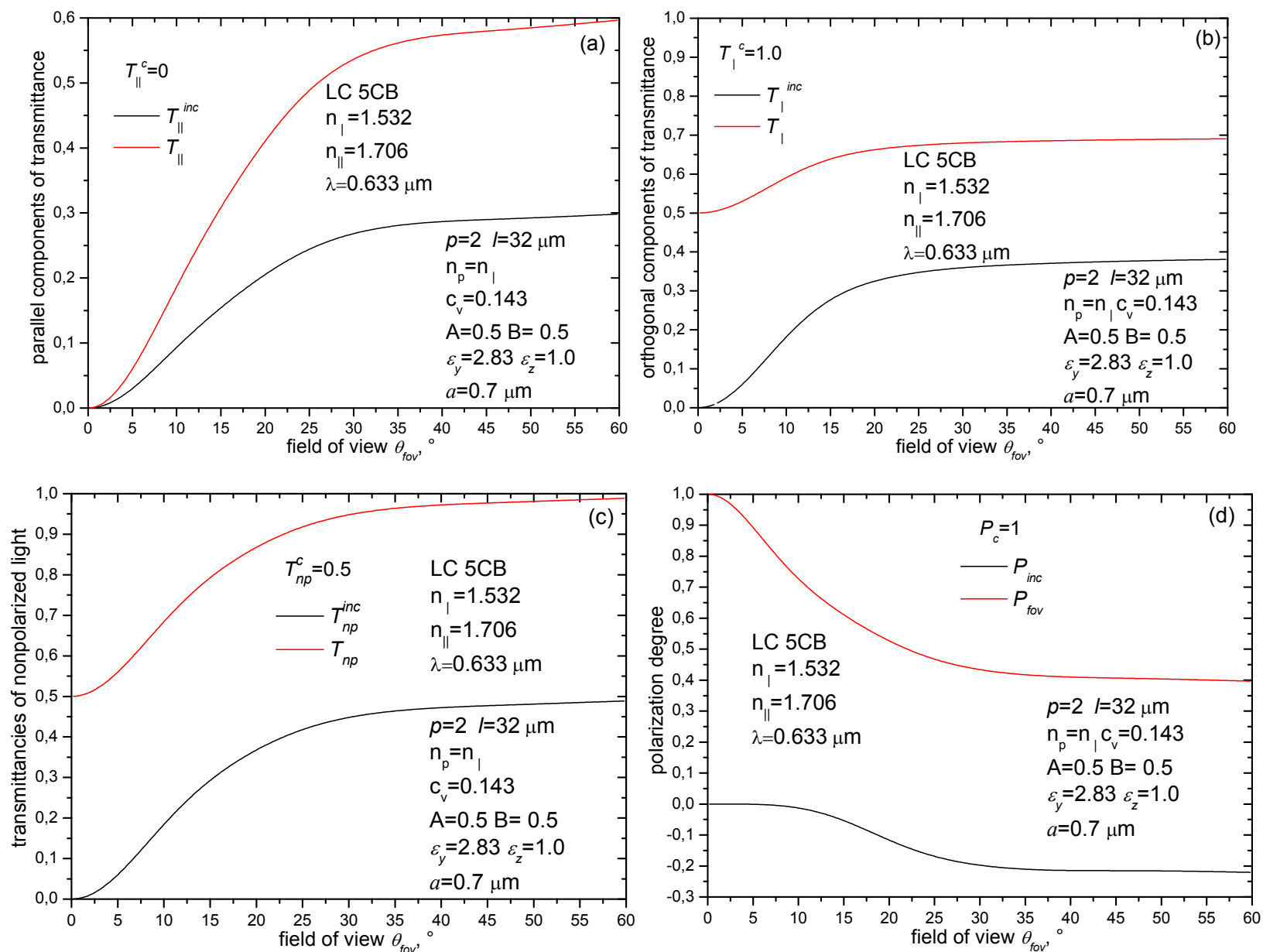

Figure 17. The dependences of the transmittance for parallel (a) and orthogonal (b) components of scattered light, the transmittance for unpolarized incident light (c), and the polarizing degree (d) on the field of view angle $\theta_{\text {fov }}$. The structure within LC droplets is monodomain. The stretching ratio of the film is $p=2 . n_{p}=n_{\perp}=1.532$. The wavelength of incident light $\lambda=0.633 \mu \mathrm{m} . a=0.7 \mu \mathrm{m}$.

The simulations were carried out for the film containing a monodisperse oriented LC droplets with monodomain internal structure. The film parameters are indicated in the legends. At such parameters, the forward-transmitted light is entirely polarized $\left(P_{f o v}=1\right.$ at $\left.\theta_{f o v}=0\right)$, and the coherent transmittance of film is $T_{n p}^{c}=0.5$.

The obtained data show that almost all incoherently scattered light is recorded at $\theta_{f o v}=60^{\circ}$; the total transmittance is $T_{n p}\left(\theta_{f o v}=60^{\circ}\right) \approx 1$. The incoherent transmission coefficient for unpolarized incident light is $T_{n p}^{i n c} \approx 0.5$ (Fig. 17 (c)). The forward-transmitted light or light transmitted in forward hemisphere, i.e. $T^{c}+T^{\text {inc }}$ (see Eqs. (26)-(28)) is partially polarized with the polarization 
degree $P_{\text {fov }} \approx 0.4$ (Fig. 17(d)). The polarization degree of diffusely scattered light is about -0.22 that corresponds to the more stronger scattering of parallel intensity component as compared with the orthogonal one.

The polarization degree of transmitted light has the values exceeding 0.95 (Fig. 17(d)) at $\theta_{f o v}<3$. Under such conditions the stretched PDLC film with monodomain and bipolar droplet director configuration effectively polarizes light and has the transmittance $\sim 0.5$.

\section{Conclusion}

A high polarization degree (more than 0.97) can be achieved for the forward-transmitted light in the wide wavelength range (from 0.45 to $0.7 \mu \mathrm{m}$ ) both for PDLC films with monodomain and bipolar LC droplet structures and for films with inhomogeneous surface anchoring of "tangentialnormal" type. A coherent transmittance of the films with bipolar droplet configuration and inhomogeneous "tangential-normal" anchoring is smaller than that of the films with monodomain internal structure. The films with homogeneous surface anchoring and radial LC droplet structure do not allow polarizing effectively the forward-transmitted light.

The angular distributions of intensity of light scattered by the PDLC films with bipolar and monodomain internal droplet structures at the stretching ratio equaled to 2 are practically coincided. At that, the polarization degrees are the same at the different wavelengths and do not depend on the polar scattering angle when the refractive index of polymer matrix is equal to the ordinary refractive index of LC.

The stretched PDLC films with monodomain and bipolar droplet structures polarize effectively light for the small FOVs $\left(<3^{\circ}\right)$. The polarization degree can exceed 0.95 at the transmittance close to 0.5 .

The developed optical-mechanical model to analyze the coherent transmittance, angular and spectral characteristics of light scattered by the stretched PDLC film allows solving the inverse problem: to determine the structural parameters of the film (the filling factor and thickness, sizes and anisometry parameters of the droplets, refractive indices of the polymer matrix and LC) at which its transmittance and polarizing ability simultaneously reach the values close to the limit ones (0.5 and 1.0, respectively).

The obtained results can be used to develop the polarizers of transmitted light based on the stretched PDLC films with the surfactant-induced modification of surface anchoring, operating in the light-scattering mode and possessing by a high transmittance and polarizing ability.

\section{Acknowledgments}


The work was performed in the framework of an agreement on inter-academic cooperation between the National Academy of Sciences of Belarus and the Siberian Branch of the Russian Academy of Sciences and supported by the Belarusian Republican Foundation for Fundamental Research (project No. F18RA-003). M.N. Krakhalev thanks the Russian Foundation for Basic Research and Government of Krasnoyarsk Territory, Krasnoyarsk Regional Fund of Science, grant №18-42243006.

\section{References}

[1] Schurcliff WA. Polarised Light. Cambridge: Harvard Univ Press; 1962.

[2] Dirix YJL. Polarisers based on anisotropic absorbance or scattering of light. Eindhoven: Technische Universiteit; 1997.

[3] Agabekov VE, Potapov AL, Shahab SN. Polarizers based on polyvinyl alcohol and films with silver nanoparticles: manufacturing and using (a review). Polim Mater Tekhnol 2015;1(2):6-35.

[4] West JL, Doane JW, Zumer S. Patent US 4.685.771. Int.Cl. G02F 1/13. Publ. 11.08.1987.

[5] Zyryanov VY, Smorgon SL, Shabanov VF. Elongated films of polymer-dispersed liquid crystals as scattering polarizers. Molecular Engineering 1992;1:305-310.

[6] Aphonin OA, Panina YuV, Pravdin AB, Yakovlev DA. Optical properties of stretched polymer dispersed liquid crystal films. Liquid Crystals 1993;15(3):395-497.

[7] Bloisi F, Ruocchio C, Terrecuso P, Vicari L. Optoelectronic polarizer by PDLC. Liquid Crystals 1996;20(3):377-379.

[8] Amimori I, Priezjev NV, Pelcovits RA, Crawford GP. Optomechanical properties of stretched polymer dispersed liquid crystal films for scattering polarizer applications. J of Appl Phys 2003: 93(6):3248-3252.

[9] K£osowicz SJ, Aleksander M. Effect of polymer-dispersed liquid crystal morphology on its optical performance. Opto-Electronics Review 2004;2(3):305-312.

[10] Smorgon SL., Presnyakov V.., Zyryanov VYa, Shabanov VF. Device for polarization and modulation of light. Instruments and Experimental Techniques (in Rus.) 1997, №1:164.

[11] Krakhalev MN, Loiko VA, Zyryanov VYa. Electro-optical characteristics of polymer-dispersed liquid crystal film controlled by ionic-surfactant method. Technical Physics Letters 2011;37(1):34-36.

[12] Prishchepa OO, Egamov MKh, Gerasimov VP, Krakhalev MN, Loiko VA. Light Polarizers Based on Composite "Polymer-LC- Surfactant" Films as Anisotropically Scattering Media. Russian Physics Journal (in Rus.) 2013;56(2/2):257-263.

[13] Egamov MKh, Gerasimov VP, Krakhalev MN, Prishchepa OO, Zyryanov VYa, Loiko VA. Polarizing properties of a stretched film of a polymer-dispersed liquid crystal with a surfactant dopant. J Opt Technol 2014;81:414-417.

[14] Loiko VA, Konkolovich AV, Zyryanov VYa, Miskevich AA. Polarization of Light by a Polymer Film Containing Elongated Drops of Liquid Crystal with Inhomogeneous Interfacial Anchoring. Opt and Spectr 2017;122(6):984-994.

[15] Ishimary A. Wave Propadation and Scattering in Random Media. New York, San Francisco, London: Academic; 1978. 
[16] Loiko VA, Konkolovich AV. Phase Change of a Plane Wave Propagating Through a Polymer Film with Encapsulated Nanodimensional Nematic Liquid Crystal Particles. JETP 2003;96(3):489-495.

[17] Loiko VA, Konkolovich AV. Polarization of Light Transmitted through a Polymer Film with Nanosized Droplets of Liquid Crystal. JETP 2004;99(2):343-351.

[18] Loiko VA. Polymer Films with Nanosized Liquid-Crystal Droplets: Extinction, Polarization, Phase, and Light Focusing. In: Wang ZM, editor. Nanodroplets. New York, Heidelberg, Dordrecht, London: Springer; 2013. p. $195-235$.

[19] Loiko VA, Zyryanov VYa. Konkolovich AV, Miskevich AA. Light Transmission of Polymer-Dispersed Liquid Crystal Layer Composed of Droplets with Inhomogeneous Surface Anchoring. Opt and Spectr 2016;120(1):143-152.

[20] Bohren CF, Huffman DR. Absorption and Scattering of Light by Small Particles. New York: Wiley; 1983.

[21] Loiko VA, Maschke U, Zyryanov VYa, Konkolovich AV, Miskevich AA. Angular Structure of Light Scattered by Monolayer of Polydisperse Droplets of Nematic Liquid Crystal. Opt and Spectr 2011;110(1):110118.

[22] Loiko VA, Konkolovich AV, Zyryanov VYa, et. al. Small-Angle Light Scattering Symmetry Breaking in Polymer-Dispersed Liquid Crystal Films with Inhomogeneous Electrically Controlled Interface Ahchoring. JETP 2017; 124:388-405.

[23] Loiko VA, Miskevich AA. Multiple Scattering of Light in Ordered Particulate Media. In: Kokhanovsky AA, editor. Springer Series in Light Scattering. Vol. 1, Multiple Light Scattering, Radiative Transfer and Remote Sensing. Springer International Publishing; 2018. p. 101-230.

[24] Loiko VA, Konkolovich AV, Miskevich AA. Light Scattering by a Nematic Liquid Crystal Droplet: WentzelKramers-Brillouin Approximation. JETP 2016;122(1):176-192.

[25] Zumer S. Light scattering from nematic droplets: Anomalous-diffraction approach. Phys Rev A 1988;37:40064015.

[26] Loiko VA, Maschke U, Zyryanov VYa, Konkolovich AV, Miskevich AA. Coherent transmission and angular structure of light scattering by monolayer films of polymer dispersed liquid crystals with inhomogeneous boundary conditions. Opt and Spectr 2011;111(6):866-872.

[27] Loiko VA, Zyryanov VYa, Maschke U, Konkolovich AV, Miskevich AA. Small-angle light scattering and transmittance of polymer film, containing liquid crystal droplets with inhomogeneous boundary conditions. JQSRT 2012;113:2585-2592.

[28] Loiko VA, Krakhalev MN, Konkolovich AV, Prishchepa OO, Miskevich AA, Zyryanov VYa. Experimental results and theoretical model to describe angular dependence of light scattering by monolayer of nematic droplets. JQSRT 2016;178:263-268.

[29] Li J, Wen C-H, Gauza S, Lu R, Wu S-T. Refractive Indices of Liquid Crystals for Display Applications. IEEE 2005;1(1):51-61. 